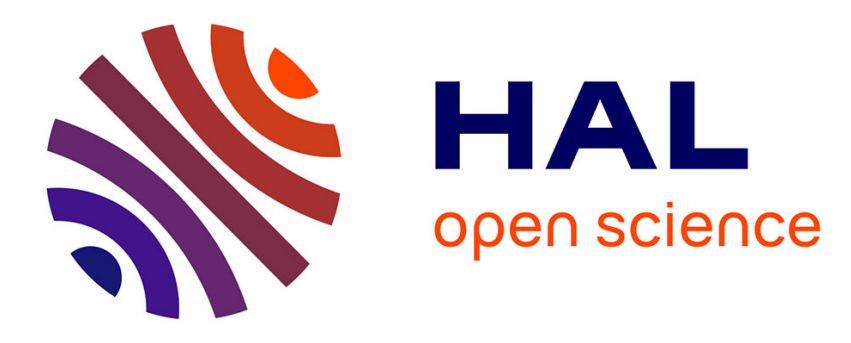

\title{
Determination of the critical exponents in the Ag Mn spin glass
}

H. Bouchiat

\section{To cite this version:}

H. Bouchiat. Determination of the critical exponents in the Ag Mn spin glass. Journal de Physique, 1986, 47 (1), pp.71-88. 10.1051/jphys:0198600470107100 . jpa-00210186

\section{HAL Id: jpa-00210186 https://hal.science/jpa-00210186}

Submitted on 1 Jan 1986

HAL is a multi-disciplinary open access archive for the deposit and dissemination of scientific research documents, whether they are published or not. The documents may come from teaching and research institutions in France or abroad, or from public or private research centers.
L'archive ouverte pluridisciplinaire HAL, est destinée au dépôt et à la diffusion de documents scientifiques de niveau recherche, publiés ou non, émanant des établissements d'enseignement et de recherche français ou étrangers, des laboratoires publics ou privés. 
Classification

Physics Abstracts

$75.30 \mathrm{H}-75.30 \mathrm{~K}$

\title{
Determination of the critical exponents in the AgMn spin glass
}

\author{
H. Bouchiat \\ Laboratoire de Physique des Solides, Bât. 510, 91405 Orsay, France
}

(Reçu le 28 juin 1985, accepté le 3 septembre 1985)

\begin{abstract}
Résumé. - Nous présentons des mesures d'aimantation non linéaire dans le système verre de spin AgMn couvrant une large gamme de concentrations $(0,4 \%<c<24 \%)$. L'analyse des résultats dans le cadre de l'existence d'une transition de phase à $T_{\mathrm{g}}$ conduit aux valeurs $\delta=3,1 \pm 0,2, \gamma=2,2 \pm 0,2$ et $\beta=1 \pm 0,1$. Ces exposants sont obtenus à partir de trois déterminations expérimentales indépendantes dans le domaine de température et de champ magnétique où $\left(T-T_{\mathrm{g}}\right) / T_{\mathrm{g}}<0,1$ et où l'aimantation non linéaire est inférieure au dixième de l'aimantation totale. A l'extérieur de ce domaine on observe systématiquement des déviations vers des valeurs apparentes plus élevées pour les exposants critiques. Ces observations expliquent le désaccord entre nos résultats et ceux obtenus sur le système CuMn [5]. L'analyse des équations de champ moyen Sherrington Kirkpatrick [17] montre en effet que la présence de termes réguliers entâche d'erreur la détermination des exposants critiques lorsque $\left(T-T_{\mathrm{g}}\right) / T_{\mathrm{g}}>0,1$ et $H>0,1$. Ce point de vue permet aussi d'apporter quelques éclaircissements concernant la diversité des exposants critiques déterminés sur d'autres systèmes.
\end{abstract}

\begin{abstract}
We present nonlinear magnetization measurements in the AgMn spin glass in a wide concentration range $(0.4 \%<c<24 \%)$. The analysis of the results in the frame work of the existence of a phase transition at $T_{\mathrm{g}}$ yields the critical exponents $\delta=3.1 \pm 0.2, \gamma=2.2 \pm 0.2$, and $\beta=1 \pm 0.1$. These exponents are obtained from three independent experimental determinations in the range of temperature and magnetic field in which $\left(T-T_{g}\right) /$ $T_{\mathrm{g}}<0.1$ and the nonlinear magnetization is less than $10 \%$ of the total magnetization. Outside this range, deviations towards higher apparent values of the critical exponents are systematically observed which explains the disagreement between our findings and those on the CuMn system [5]. The analysis of the mean-field Sherrington-Kirkpatrick equations [17] indeed shows that the presence of regular terms alters the determination of the critical exponents for $\left(T-T_{\mathrm{g}}\right) / T_{\mathrm{g}}>0.1$ and $H>0.1$. This point of view may also help to clarify the diversity existing between the values of the critical exponents determined in other systems.
\end{abstract}

\section{Introduction.}

Since the singular behaviour of the nonlinear magnetization has been experimentally demonstrated in a spin glass system [1-3] great amount of experimental work has been devoted to this subject analysing the nonlinear magnetization in several systems with a scaling function [4 to 9]. However the critical exponents strongly differ from one system to another. On the theoretical side the nature of the spin glass transition and even its existence in three dimensions are still very controversial : perturbation analyses of the mean field theory below $d=6$ seem to indicate [10] that $d=4$ is the lowest critical dimension. Furthermore numerical simulations performed on bidimensional short-range Ising systems with a $T=0$ transition claimed to reproduce some of the experimental results analysed in the framework of a finite temperature phase transition [11 to 14]. However more recent simulations in three dimensional systems $[15,16]$ are consistent with a finite temperature phase transition. We think that experiments can shed light on these ambiguities when performed in a range of temperatures and magnetic fields where regular contributions in the nonlinear magnetisation (i.e. diverging at $T=0$ ) cannot be mistaken with singular terms (i.e. diverging at $T=T_{\mathrm{g}}$ ).

In this paper we present nonlinear magnetization measurements on the AgMn spin glass with concentrations between $0.4 \%$ to $20 \%$. Unlike what is done in the study of classical phase transitions the purpose of this work is not to measure critical exponents with 
an accuracy of $10^{-3}$, but to explore the AgMn spin glass system in a wide concentration range with as many as possible independent experimental criteria, yielding information on the critical exponents. In doing so we do not aim at an accuracy better than $10 \%$ on the values of the critical exponents but rather we seek an internal consistency between the different criteria used for the various samples. Furthermore we want to check to what extent the measured values of the critical exponents depend on the range of field and temperature studied for their determination, either because of the existence of regular terms, or because of the existence of crossovers between different types of critical behaviour. This point may explain the discrepancies existing between the different values of these exponents determined so far by different authors [1 to 9].

The paper is organized as follows :

In Section 1 we present an " experimental » analysis of the mean field solution of the Sherrington Kirkpatrick [17] model above $T=T_{\mathrm{g}}$ calculated numerically. Knowing the values of the critical exponents for this problem we can illustrate how the presence of regular terms in the nonlinear magnetization makes it impossible to determine these exponents correctly out of the very vicinity of the critical point.

In Section 2 we describe our experimental set up which enables us to measure exclusively the nonlinear magnetization as a function of the magnetic field by compensating out the linear part of it.

In Section 3 we explain our method of analysis of the nonlinear magnetization. We have used three independent experimental criteria to determine the critical exponents among which two only are independent.

When our analysis is restricted to the temperature range $\left|\frac{T-T_{\mathrm{g}}}{T_{\mathrm{g}}}\right|<0.1$ and to the range of magnetic field such that $M_{\mathrm{NL}} / M<0.1$, we find $\gamma=2.2 \pm 0.2$, $\delta=3 \pm 0.2$, and $\beta=1 \pm 0.1$ for samples with concentrations varying between 0.4 at. $\%$ and 20 at. $\%$. It is possible to describe our data with a universal scaling function compatible with these exponents. However we show that our data in the range of temperature $0.1<\frac{T-T_{\mathrm{g}}}{T_{\mathrm{g}}}<0.4$ deviates from this scaling behaviour. We finally complete this analysis by pointing out properties of the temperature dependence of the magnetization itself which can be related to the values of the critical exponents and thus independently confirm our determinations.

In Section 4 we discuss to what extent our data can rule out $T_{\mathrm{c}}=0$ phase transition conjectures. We finally discuss the disagreement between our values of the critical exponents and those determined on CuMn [4] in the temperature range $0.1<\frac{T-T_{\mathrm{g}}}{T_{\mathrm{g}}}<1$. We wonder whether this has to be attributed to regular terms or to crossover between different types of critical behaviours.
1. Scaling analysis of the mean field equations : an experimental approach.

Before analysing the singular behaviour of the nonlinear magnetization in AgMn with critical exponents and universal scaling functions, let us discuss the range of validity of such an analysis on the mean field solution [17]. In the following we consider the selfconsistent equations of Sherrington and Kirkpatrik which describe a system of $N$ Ising spins with in finite range random interactions of variance $J / \sqrt{N}$. The order parameter is related to the average square of the local magnetization $q=\overline{\left\langle S_{i}\right\rangle^{2}}(\langle\rangle$ denotes thermal average, — denotes average on disorder)

$$
\begin{aligned}
& q=\frac{1}{\sqrt{2 \pi}} \int_{-\infty}^{+\infty} \exp \left(-\frac{1}{2} z^{2}\right) \operatorname{th}^{2} \frac{1}{T}\left(J q^{1 / 2} z+H\right) \mathrm{d} z \\
& M=\frac{1}{\sqrt{2 \pi}} \int_{-\infty}^{+\infty} \exp \left(-\frac{1}{2} z^{2}\right) \operatorname{th} \frac{1}{T}\left(J q^{1 / 2} z+H\right) \mathrm{d} z .
\end{aligned}
$$

These equations yield a phase transition at $T_{\mathrm{g}}=J$, however, they do not properly describe the low temperature phase as has been shown by De Almeida and Thouless [18]. The correct solution involves an infinite number of order parameters describing the degeneracy of the free energy ground states [19]. Equations (1) and (2) properly describe the mean field solution for $T>T_{\mathrm{g}}$. Note the coupling between the order parameter and the magnetization in equation (2).

Equation (1) describes the coupling of the spin glass order parameter with a uniform magnetic field $H$, involving even powers of $H$. The equivalent of the magnetic field in a classical ferromagnetic transition (which favours one of the low energy states) is here, indeed a staggered field being fixed in time but random in direction on each spin site. One might think that a uniform magnetic field is not coupled to the order parameter, just as in a pure antiferromagnetic system. This is true when one considers the linear term of the magnetization which follows the paramagnetic Curie law down to $T=T_{\mathrm{g}}$. But in contrast with the pure antiferromagnetic case, where above the Neel temperature the exact compensation of the two sublattices exactly cancels the molecular field at each site, the disorder inherent to spin glasses makes these cancellations impossible. The molecular field is thus a random variable whose variance is $J^{2} \overline{\left\langle S_{i}\right\rangle^{2}}=J^{2} q$ (Eq. (3)).

Equations (1) and (2) exactly express this point when written like :

$$
q=\frac{1}{\sqrt{2 \pi J^{2} q}} \int_{-\infty}^{+\infty} \exp \left(-\frac{x^{2}}{2 J^{2} q}\right) \operatorname{th}^{2} \frac{1}{T}(x+H) \mathrm{d} x
$$

$$
M=\frac{1}{\sqrt{2 \pi J^{2} q}} \int_{-\infty}^{+\infty} \exp \left(-\frac{x^{2}}{2 J^{2} q}\right) \text { th } \frac{1}{T}(x+H) \mathrm{d} x
$$


The nonlinear part of $M$ couples to the order parameter. In the development of the magnetization in successive powers of the magnetic field, every term of degree strictly above one is singular [20] and contains the critical exponents of the spin glass phase transition [21]. To lowest order, in $H /\left(T-T_{g}\right)$ equation (1) yields tor the order parameter :

At $T>T_{\mathrm{g}}$

$$
\begin{gathered}
q=\frac{H^{2}}{T^{2}-T_{\mathrm{g}}^{2}}+0\left(H /\left(T-T_{\mathrm{g}}\right)\right)^{4} \\
T=T_{\mathrm{g}} ; q=\frac{H}{\sqrt{2} T_{\mathrm{g}}}+0\left(H^{2}\right) .
\end{gathered}
$$$$
\text { at }
$$

The nonlinear magnetization is coupled to $q$ via the relation :

$$
M=\frac{H}{T}-\frac{1}{3} \frac{H}{T^{3}}\left(3 J^{2} q+H^{2}\right)
$$

valid to third order in $H$. Using equation (5) for $q$ we find : (i.e. at $T>T_{\mathrm{g}}$ and $H \ll T-T_{\mathrm{g}}$ )

$$
M=\frac{H}{T}-\frac{1}{3} \frac{H^{3}}{T^{3}} \frac{T^{2}+2 T_{\mathrm{g}}^{2}}{\left(T^{2}-T_{\mathrm{g}}^{2}\right)}
$$

the nonlinear magnetization diverges as $\frac{1}{T-T_{\mathrm{g}}}$ in the neighbourhood of $T_{\mathrm{g}}$.

At $T=T_{\mathbf{g}}$

$$
M=\frac{H}{T_{\mathrm{g}}}-\frac{H^{2}}{\sqrt{2} T_{\mathrm{g}}^{2}} .
$$

Equations (1) and (2) can be easily solved for any values of $T-T_{\mathrm{g}}$ and $H$ by using a microcomputer yielding the complete equation of state $M(H, T)$ in this model.

In order to estimate the range of validity of the scaling laws in this particular case, let us compare $M(H, T)$ to the form expected in the framework of the scaling hypothesis.

$$
\begin{gathered}
M_{\text {sc. }}(H, T)=\frac{H}{T}\left[1-\tau^{\beta} f\left(h^{2} / \tau^{\phi}\right)\right] \\
\tau=\left(T-T_{\mathbf{g}}\right) / T_{\mathbf{g}} \quad h=H / T_{\mathbf{g}}
\end{gathered}
$$

$\phi$ and $\beta$ are the critical exponents which are known to be $\phi=\gamma+\beta=2$ and $\beta=1$ in the present mean field case, $f$ is a function of the variable $x=H^{2} / \tau^{2}$ verifying :

$\lim _{x \rightarrow 0} f(x)=x$ and $\quad \lim _{x \rightarrow \infty} f(x)=x^{1 / \delta}=x^{1 / 2}$.

We have plotted the computed values of $(M(H, T)-$ $H / T) / \tau$ versus $h^{2} / \tau^{\phi}$ in figure 1 . For $0.01<h<0.1$ and $0.01<\tau<0.1$ the points superimpose on a unique curve whose asymptotic limits indeed satisfy relation (10). However for $0.1<\tau<1$ and $0.1<h<1$ the points are widely scattered. We can show that this dispersion comes from the regular terms in $M(H, T)$ which cannot be described by the scaling form $M_{\text {sc. }}$. $(H, T)$.

The first terms in the development of $M(H, T)$ in the successive powers of field yield [22] :

$$
M(H, T)=\frac{H}{T}\left(1+a(T) h^{2} / \tau+b(T) h^{4} / \tau^{3}\right)
$$

with

$$
a(T)=\frac{1}{3} \frac{T_{\mathrm{g}}}{T} \frac{T^{2}+2 T_{\mathrm{g}}^{2}}{T^{2}+T_{\mathrm{g}} T}
$$

and

$$
\begin{aligned}
b(T)= & \frac{2}{15}\left(\frac{T_{\mathrm{g}}}{T}\right) \frac{1}{\left(T^{2}+T_{\mathrm{g}} T\right)^{3}} \times \\
& \times\left[T^{6}+12 T^{4} T_{\mathrm{g}}^{2}+18 T^{2} T_{\mathrm{g}}^{4}-16\right] .
\end{aligned}
$$

The temperature dependences of $a$ and $b$ represent the corrections to the scaling assumption $a(T)=$ Cte $=a\left(T_{\mathrm{g}}\right)$ and $b(T)=\mathrm{Cte}=b\left(T_{\mathrm{g}}\right)$ and explain deviations from universality observed when $\left(T-T_{\mathrm{g}}\right) / T_{\mathrm{g}}>0.1$. Furthermore it is important to note that the change of scaling variables proposed by Souletie et al. [5, 23] cannot absorb these temperature dependences. Indeed the development of $M(H, T)$ versus $h^{\prime}=H / T$ and $\tau^{\prime}=\left(T-T_{\mathrm{g}}\right) / T$ yields :

$$
M(H, T)=\frac{H}{T}\left[1+a(T) \frac{h^{\prime 2}}{\tau^{\prime}}+b^{\prime}(T) \frac{h^{\prime 4}}{\tau^{\prime 3}}+\cdots\right]
$$

where $a^{\prime}$ and $b^{\prime}$ are temperature dependent :

$$
\begin{aligned}
& a^{\prime}(T)=\frac{1}{3} \frac{T^{2}+2 T_{\mathrm{g}}^{2}}{T^{2}+T_{\mathrm{g}} T} ; \quad a^{\prime}\left(T_{\mathrm{g}}\right)=1 / 2 ; \quad a^{\prime}(\infty)=1 / 3 \\
& b^{\prime}(T)=\frac{2}{15} \frac{\left[T^{6}+12 T^{4} T_{\mathrm{g}}^{2}+18 T^{2} T_{\mathrm{g}}^{4}-16\right]}{\left(T^{2}+T_{\mathrm{g}} T\right)^{3}} \\
& b^{\prime}\left(T_{\mathrm{g}}\right)=\frac{1}{4} ; \quad b^{\prime}(\infty)=\frac{2}{15}
\end{aligned}
$$

Figure 2a illustrates this point by showing that the $\log -\log$ plot of $a^{\prime}(T)$ versus $\tau^{\prime}$ for $\tau^{\prime}>0.1$ yields an apparent value of $\gamma: \gamma_{\text {eff }}=1.3$ which is larger than the known value of 1 obtained for $\tau^{\prime}<0.1$.

Figure $2 \mathrm{~b}$ also illustrates the error in the determination of $\delta$ (more than $30 \%$ ) which can be made by considering (in the S.K. model) the nonlinear magnetization at $T=T_{\mathrm{g}}$ in the range of field where the nonlinear part of the magnetization $M_{\mathrm{NL}}$ is more than $10 \%$ of the linear part. Indeed at $T=T_{\mathrm{g}}$ :

$$
\frac{M_{\mathrm{NL}}}{H}=\frac{H}{\sqrt{2}}-\frac{23}{24} H^{2}+O\left(H^{3}\right) .
$$

The $H^{2}$ and higher order terms are responsible for the high field deviations from the initial $H$ dependence of $M_{\mathrm{NL}} / H$ corresponding to $\delta \neq 2$. We emphasize that one can obtain an apparent good scaling of the 


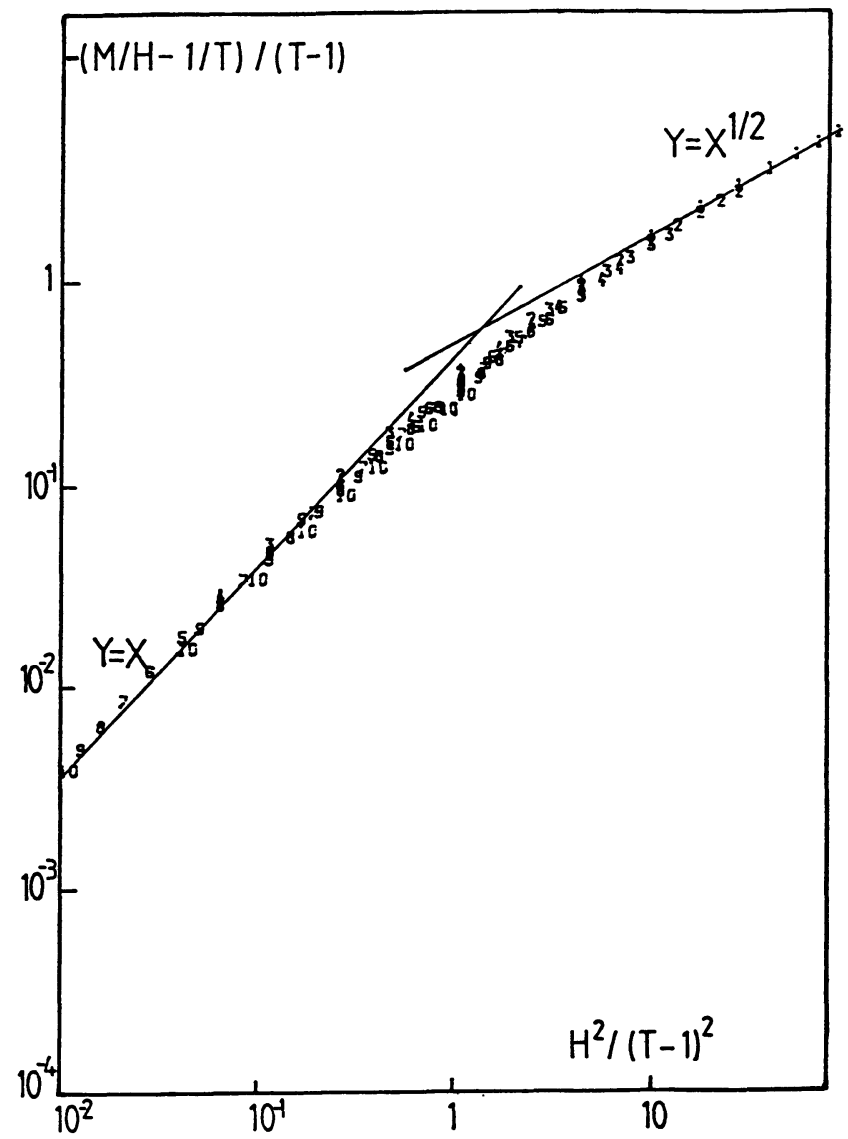

Fig. 1. - Scaling of the S.K. solution (in units where $\left.T_{\mathrm{g}}=1\right)$ a) In the range of temperature and magnetic field where $0.01<H<0.1$ and $0.01<\tau<0.1: 1: \tau=0.01$; $H=0.01,0.02, \ldots, 0.1,2: \tau=0.02 ; H=0.01, \ldots, 0.1$, etc.

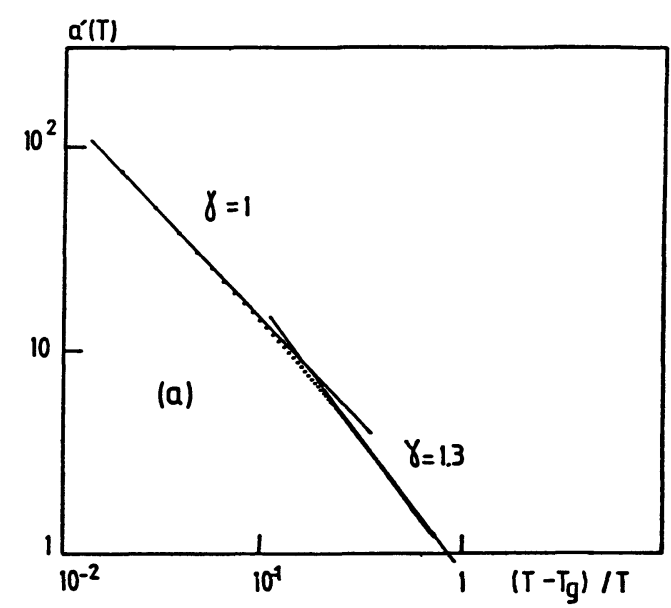

Fig. 2a. - Variation with temperature of the cubic term of nonlinear magnetization in the S.K. solution. Note deviations towards higher apparent values of $\gamma$ in the range of temperature where $\tau>0.1$.

non linear magnetization in the range of temperature $T-1>0.1$ and $H>0.1$, using $h^{\prime}$ and $\tau^{\prime}$ variables but with the wrong values $\gamma_{\text {eff }}=1.3$ and $\delta_{\text {eff }}=2.7$, for the critical exponents, see figure 3.

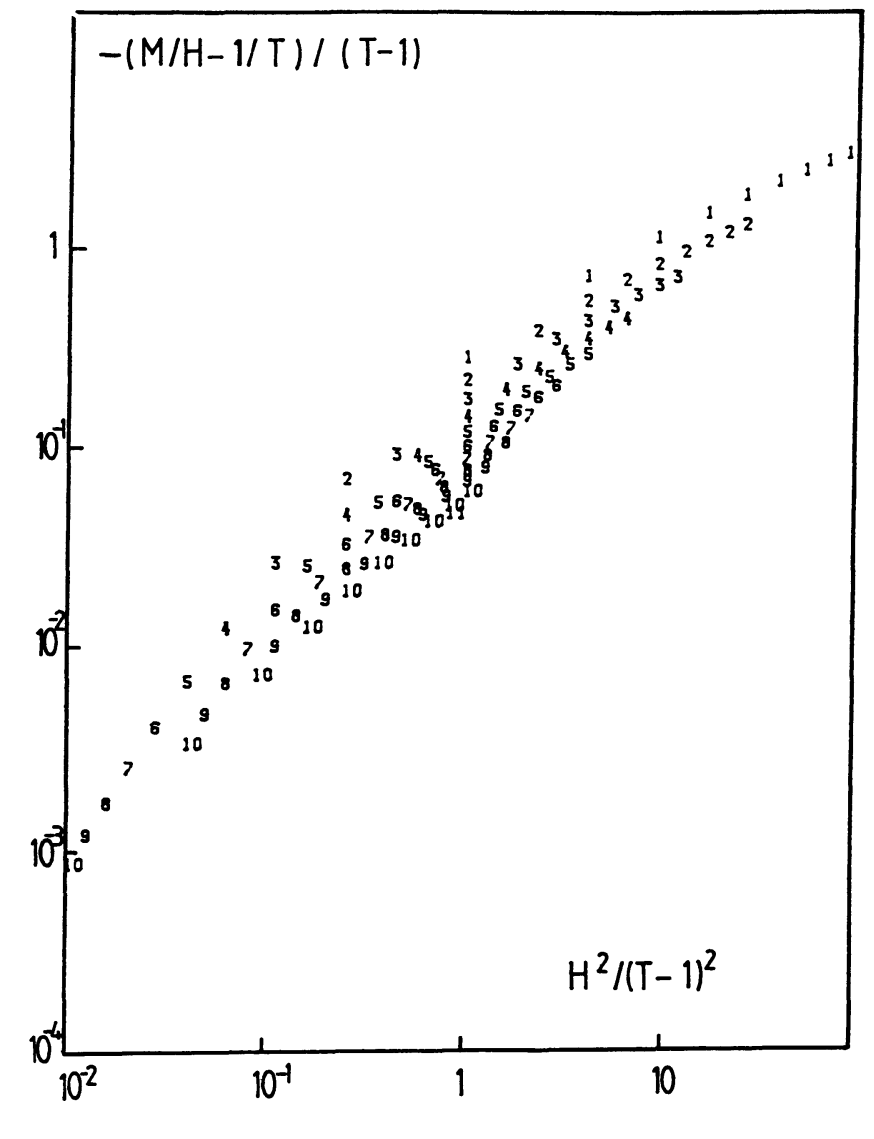

b) In the range of temperature and magnetic field where : $0.1<H<1$ and $0.1<\tau<1: 1: \tau=0.1 ; H=0.1,0.2, . ., 1$, $2: \tau=0.2 ; H=0.1, \ldots, 1$, etc.

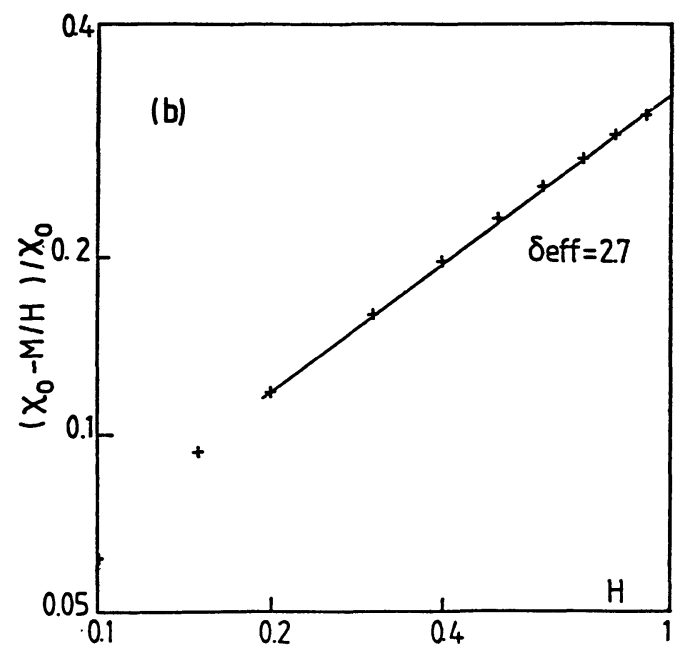

Fig. 2b. - Field dependence of the nonlinear magnetization at $T_{\mathrm{g}}$, in the range of magnetic field where $M_{\mathrm{NL}} / M>0.1$ note the apparent value of $\delta \simeq 2.7$.

In appendix I we complete this analysis with a comparison with the mean field ferromagnetic case in which the choice of $\tau^{\prime}$ and $h^{\prime}$ as scaling variables is better justified. 


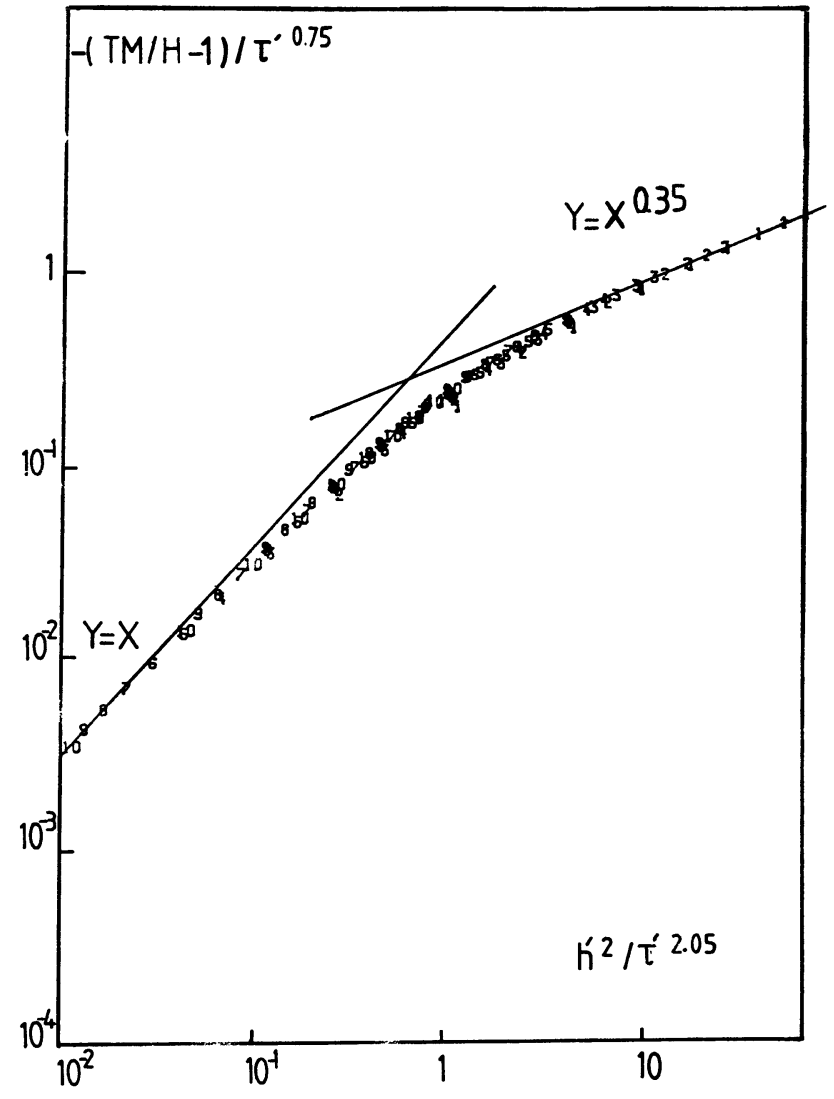

Fig. 3. - Scaling of the S.K. solution using scaling variables $\tau^{\prime}=(T-1) / T$ and $h^{\prime}=H / T$ in the range of temperature $1.1<T<2$ and $0.1<H<1$. An apparent good scaling is obtained with the wrong values of the exponents $\gamma=1.3$ and $\delta=2.7$ obtained from figure 2 .

The conclusion of this "experimental» study of the mean field solution is that the presence of regular terms in the development of the magnetization makes it impossible to determine the critical behaviour of the singular terms correctly when the regular terms are comparable to the singular terms. Taking this analysis as a rough guide we have aimed at a $10 \%$ accuracy on the critical exponents. This is the reason why we have chosen to restrict the range of our analysis to the temperature $\left(T-T_{\mathrm{g}}\right) / T_{\mathrm{g}}<0.1$ in our determination of the critical exponents. The same kind of restriction must be made concerning the choice of the range of magnetic fields. We have chosen to limit this range to the fields for which the nonlinear magnetization is less than $10 \%$ of the linear magnetisation, at $T_{\mathrm{g}}$. We think that this range of field and temperature is indeed the best choice we can do because the spin glass transition temperature is not defined to better than $\pm 0.5 \%$. (At temperatures $\left(T-T_{\mathrm{g}}\right) / T_{\mathrm{g}}<0.01$ and in very low fields one may indeed expect some rounding of the transition to occur.)

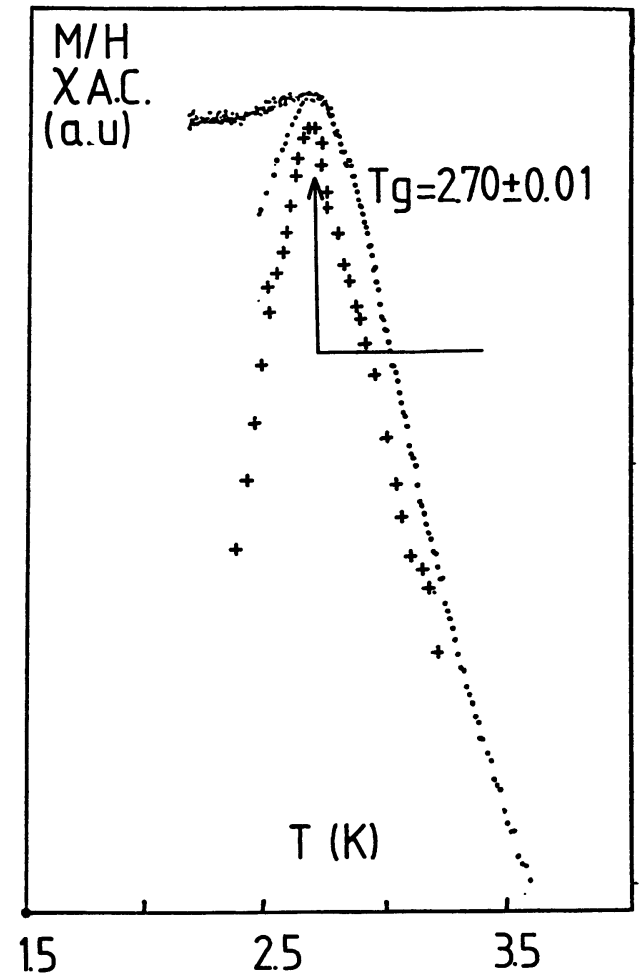

Fig. 4. - Determination of $T_{\mathrm{g}}$ on the AgMn 0.5 at. \% sample : + AC susceptibility at $32 \mathrm{~Hz}$, the cusp is found at $T=2.71 \pm 0.01 \mathrm{~K}$. D.C. magnetization measured in a 15 gauss field, the maximum takes place at $2.70 \pm 0.01 \mathrm{~K}$, the irreversibility occurs at $2.71 \pm 0.01 \mathrm{~K}$.

\section{Experimental procedure.}

The samples studied are bulk cylinders of AgMn ( $8 \mathrm{~mm}$ high and $6 \mathrm{~mm}$ wide) whose $\mathrm{Mn}$ concentrations are equal to $0.4,0.5,0.7$ and 20 at. $\%$, respectively. The 0.4 and 0.7 at. \% polycrystalline samples were obtained by melting the constituants in an induction furnace. The 0.5 and 20 at. \% samples are single crystals grown by the Bridgmann furnace method [24]. No difference concerning the sharpness of the transition could be detected between samples of different metallurgical origins. The transition temperature was evaluated by :

1) Position of the cusp in a.c. susceptibility $(f=30 \mathrm{~Hz})$ versus temperature.

2) Position of the maximum in the low field D.C. magnetization versus temperature. See figure 4.

3) Occurrence of irreversibility in the low field D.C. magnetization (i.e. the temperature below which the field cooled magnetization differs from the zero field cooled magnetization).

These three criteria for $T_{\mathrm{g}}$ coincided within $\pm 0.5 \%$ which is the error bar we attributed to the value of $T_{g}$. This result is in agreement with the very slight frequency dependence of the susceptibility cusp in 
AgMn reported in [25]. The temperature was stabilized within better than $0.1 \%$ by regulating the pressure of either a helium bath for the low concentration samples or a nitrogen bath $63 \mathrm{~K}<T<77 \mathrm{~K}$ for the 20 at. $\%$ sample $\left(T_{\mathrm{g}}=68.5 \mathrm{~K}\right)$.

The magnetization measurements were made with a vibrating sample Foner magnetometer whose sensitivity is $10^{-6} \mathrm{emu}$. A pair of Helmholtz coils produces a magnetic field between 0 and $1.5 \mathrm{~K}$ gauss homogeneous within $10^{-4}$ in a $1 \mathrm{~cm}^{3}$ sphere. The earth' magnetic field is compensated for. The measure of the current in the Helmholtz coils with a high precision shunt yields an accuracy and a reproducibility on the field measurement better than $10^{-4}$. This last point is crucial for a precise subtraction of the field linear part of the magnetization.

Furthermore in order to increase our accuracy concerning the nonlinear part of the magnetization we have compensated in situ the linear part using the following procedure depicted in figure $5:$ a small coil $(6 \mathrm{~mm}$ diameter 20 turns) is mounted on the sample holder two centimeters above the spin glass sample. This coil is biased with a current proportional to the current in the Helmholtz coils (i.e. the magnetic field), so as to produce a magnetic dipole, proportional to the field, opposite in sign to the magnetization of the sample. In this way we are able, by suitable adjustment to nearly compensate out the field linear contribution of the sample magnetization [26]. This

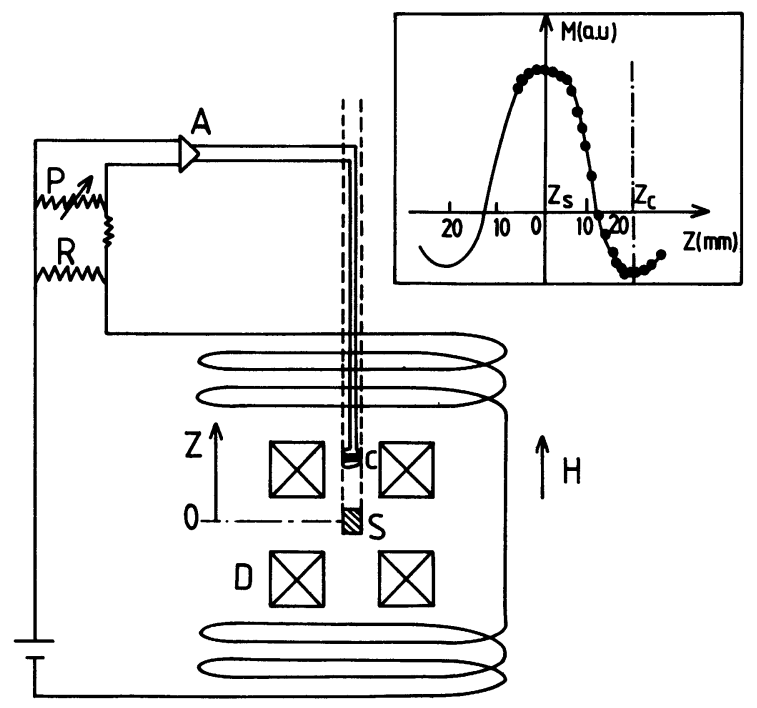

Fig. 5. - Schematic diagram of the compensation of the linear part of the magnetization. $H$ : Helmholtz coils; D : Pickup coils ; C : compensation coil ; S : Sample; R : high precision shunt $\sim(0.04 \Omega) ; P$ : Potentiometer adjusting the current in the compensating coil; $A$ : Current amplifier. Insert : response of the pickup coils versus the position of the sample along the vertical axis. Zs denotes the proper position of the sample, Zc denotes the proper position of the compensating coil centred in the secondary lobe of the pick up coils response function at a distance of the sample $(2 \mathrm{~cm})$ such as to produce a negligible perturbation of the applied magnetic field on the sample. procedure enables us to measure nonlinear contributions which are less than $10^{-3}$ of the linear part of the magnetization. Data acquisition and magnetic field runs are automatized via a microcomputer.

Typical sets of curves yielding the variation with magnetic field of $\Delta M / H$ for different temperatures near $T_{\mathrm{g}}$ are shown in figure 6 ( $\Delta M$ denotes the magnetization of the spin glass sample whose linear part has been compensated as above). At temperatures above $1.1 T_{\mathrm{g}} \Delta M / H$ is quadratic in low magnetic fields as expected for a paramagnet. At lower temperatures the curvature of $\Delta M / H$ versus field increases drastically until $T=T_{\mathrm{g}}$ where the low field behaviour of $\Delta M / H$ is singular (i.e. cannot be described by an analytic function of $H^{2}$ ).

In the following we discuss the analysis of this singularity in terms of critical exponents and a scaling function.

\section{Critical analysis of the nonlinear magnetization.}

3.1 DETERMINATION OF $\delta$ FROM THE NONLINEAR MAGNETIZATION AT $T_{\mathrm{g}}$ - - In figure 7 the nonlinear magnetization divided by the magnetic field is plotted on a logarithmic scale, for the 0.5 at. $\%$ at $T=T_{\mathrm{g}}=2.70 \mathrm{~K}$.

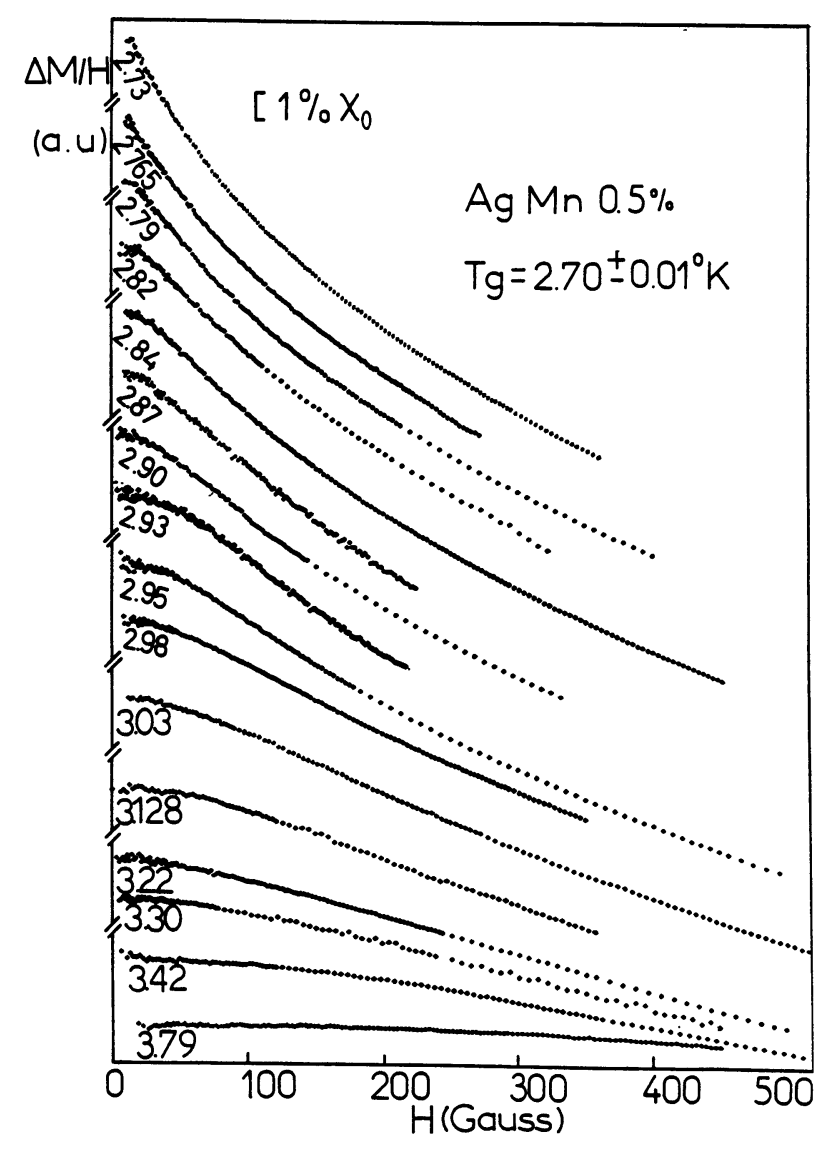

Fig. 6. - Typical set of curves yielding $\Delta M / H$ as the function of the magnetic field at different temperatures, above the transition temperature, for the 0.5 at. $\%$ sample. The relative origins on the $Y$ axis are arbitrary. $\chi_{0}$ denotes the value of the linear magnetization per unit field at $T=T_{\mathrm{g}}$. 


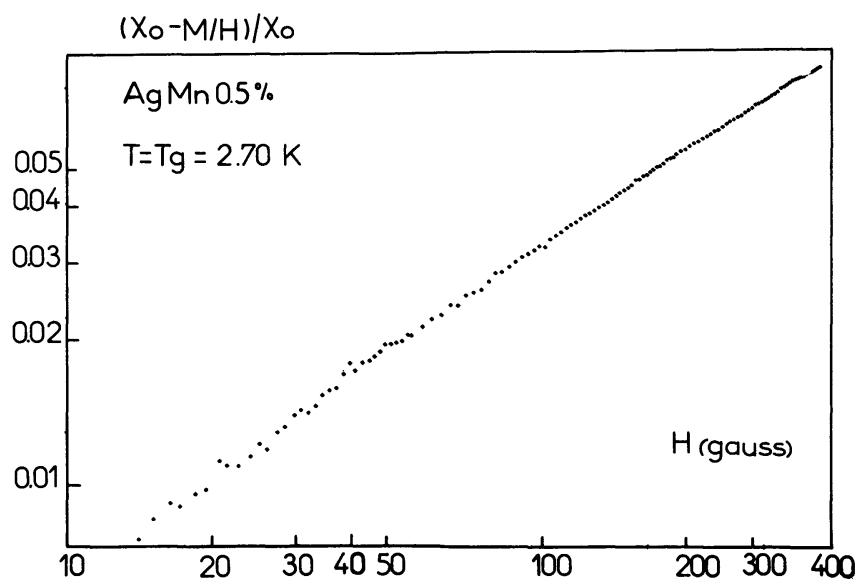

Fig. 7. - Log-Log plot of the non linear magnetization versus magnetic field at $T_{\mathrm{g}}$ for the 0.5 at. $\%$ in the range of magnetic field where $M_{\mathrm{NL}} / M<0.1$, the slope yields $2 / \delta=$ $0.64 \pm 0.02$.

It is well fitted between 10 gauss and 400 gauss by a power law :

$$
\begin{gathered}
M_{\mathrm{NL}} / H \propto H^{2 / \delta} \\
2 / \delta=0.64 \pm 0.02 .
\end{gathered}
$$

This yields :

$$
\delta=3.1 \pm 0.1 \text {. }
$$

The same exponent has been obtained for the other concentrations. Figure 8 shows $(M / H) / \chi_{0}$ versus $\left(H / H_{0}\right)^{0.64}$ for three different concentrations where the concentration dependence of $H_{0}$ is given in table $I$. The $H^{\mathbf{0 . 6 4}}$ law is well satisfied in the range of magnetic fields where $M_{\mathrm{NL}} / M<0.1$. Deviations towards apparent higher values of $\delta$ occur at higher field as shown

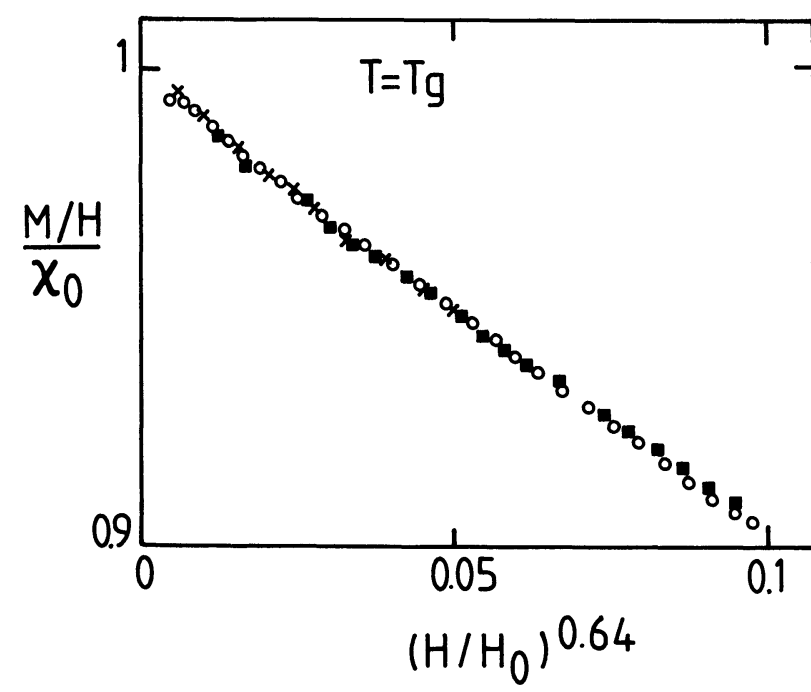

Fig. 8. $-M / H$ scaled to the linear susceptibility at $T_{\mathrm{g}}$ is plotted versus reduced magnetic field $\left(H / H_{0}\right)^{0.64}$ for three different concentrations : $\operatorname{AgMn~} 0.4 \%$; O AgMn $0.5 \%$; $\times \operatorname{AgMn} 20.5 \%$.
Table I. - Analysis of the non linear magnetization at $T_{\mathrm{g}}: M / H=\chi_{0}\left(1-\left(H / H_{0}\right)^{0.64}\right)$.

\begin{tabular}{lcc}
\hline$C$ at. $\%$ & $T_{\mathrm{g}}(\mathrm{K})$ & $H_{\mathbf{0}}(\mathrm{K}$ gauss $)$ \\
\hline 0.4 & $2.15 \pm 0.01$ & 14.8 \\
0.5 & $2.70 \pm 0.01$ & 12.3 \\
0.7 & $3.81 \pm 0.02$ & 8.88 \\
$2.6[33]$ & $10 \pm \pm 0.05$ & 29.6 \\
$10.6[2]$ & $37.4 \pm 0.2$ & 59.2 \\
20.5 & $68.5 \pm 0.3$ & 228 \\
\hline
\end{tabular}

in figure 9 on the 0.5 at. \% sample. We may attribute these deviations to the presence of higher order terms in the development of $M_{\mathrm{NL}} / H$ versus $H$ whose contribution for high values of the magnetic field is not negligible compared to the $H^{2 / \delta}$ term. The effect of such terms has already been discussed in 2 on the S.K. equations. Our determination of $\delta$ by measuring the field dependence of the nonlinear D.C. magnetization at $T=T_{\mathrm{g}}$ in a range of field where $M_{\mathrm{NL}} / M<10^{-1}$ are the first of that kind. Indeed the determination of $\delta$ on CuMn by Omari, Préjean and Souletie [5] was made in a range of fields where $0.1<M_{\mathrm{NL}}<$ $M<0.5$. This remark combined with our own observations explain the discrepancy between their determination of $\delta=5$ and our value of $\delta=3$ (see Table II).

3.2 DETERMINATION OF $\gamma$ FROM THE DIVERGENCE OF THE $H^{2}$ TERM IN $\Delta M / H$. - The initial slope $: a(T)$ of $\Delta M / H$ plotted versus $H^{2}$ yields the exponent $\gamma$. In figure 10 and 11 one can see that the range of field : $\Delta H$ where $\Delta M / H$ is linear in $H^{2}$ decreases drastically when $T \rightarrow T_{\mathrm{g}} . \Delta H^{2}$ varies approximately like $\tau^{3}$ in the range of temperatures where $\tau<0.1$. For the 20 at. \% AgMn sample we took into account the non-



Fig. 9. - Log-Log plot of the non-linear magnetization versus magnetic field at $T_{\mathrm{g}}$ for the 0.5 at. \% sample in the range of magnetic field where $M_{\mathrm{NL}} / M<0.5$. Note deviations towards apparent values of $\delta$ which are higher than the value deduced when the analysis is restricted to the range of field where $M_{\mathrm{NL}} / M<0.1$. 
Table II. - Measured values of critical exponents on various spin glass systems. Some of these values were directly measured like $\gamma$ and $\delta$ others are the result of the optimization of the scaling like $\phi$ and $\beta$.

\begin{tabular}{|c|c|c|c|c|c|c|c|c|}
\hline System & Technique & $\begin{array}{c}\text { Range } \\
\text { of temperature } \\
\left(T^{\max }-T_{\mathrm{g}}\right) / T_{\mathrm{g}}\end{array}$ & $\begin{array}{l}\text { Range of field } \\
M_{\mathrm{NL}} \max / M\end{array}$ & $\delta$ & $\phi$ & $\gamma$ & $\beta$ & Reference \\
\hline CuAlMn $(1 \% M n)$ & A.C. & & 0.15 & $2.9 \pm 0.4$ & & & & [1] \\
\hline $\operatorname{CuMn}(2 \% \mathrm{Mn})$ & A.C. & & 0.02 & $1.9 \pm 0.1$ & & & & [36] \\
\hline $\operatorname{CuMn}(4.6 \% \mathrm{Mn})$ & D.C. & 1 & 0.5 & $1.15 \pm 0.15$ & $5 \pm 0.5$ & 3.4 & 1 & [2] \\
\hline CuMn $(0.25 \% \mathrm{Mn})$ & D.C. & 0.7 & & 4.5 & & 3.5 & 1 & [4] \\
\hline CuMn (1 at. \% Mn) & D.C. & 2 & 0.5 & 4.4 & & $3.3 \pm 0.05$ & 1 & [5] \\
\hline AgMn $(10.6 \% \mathrm{Mn})$ & D.C. & 0.4 & 0.1 & & & $1.5 \pm 0.5$ & & [3] \\
\hline $\operatorname{AgMn}(0.4 \%, 0.5 \%, 0.7 \%, 20.5 \%)$ & D.C. & 0.1 & 0.1 & $3.1 \pm 0.2$ & $3.3 \pm 0.2$ & $2.2 \pm 0.2$ & $1 \quad \pm 0.1$ & this work \\
\hline $\mathrm{AuFe}(1.5 \% \mathrm{Fe})$ & A.C. $-3 \omega$ & 0.1 & 0.01 & $2 \pm 0.2$ & & $1.1 \pm 0.2$ & 0.9 & [38] \\
\hline GdAl $(37 \%$ Gd $)$ & & 1 & 0.6 & $6.1 \pm 0.2$ & $4 \pm 0.5$ & $3.8 \pm 0.5$ & & [2] \\
\hline GdAl $(37 \%$ Gd $)$ & D.C. & 0.16 & 0.3 & $5.7 \pm 0.2$ & $3.3 \pm 0.4$ & $2.7 \pm 0.1$ & & [39] \\
\hline $\mathrm{Fe}_{10} \mathrm{Ni}_{70} \mathrm{P}_{20}$ & A.C. $-3 \omega$ & 0.3 & 0.15 & $5.2 \pm 0.5$ & & $2.3 \pm 0.2$ & & [8] \\
\hline $\mathrm{Al}_{2} \mathrm{O}_{3} \mathrm{MnOSiO}_{2}(15 \% \mathrm{Mn})$ & A.C. & 0.4 & 0.05 & 3.2 & 4.5 & $3.1 \pm 0.1$ & $1.4 \pm 0.1$ & {$[7]$} \\
\hline $\mathrm{CsNiFeF}_{6}$ & D.C. & 0.1 & 0.5 & 3.5 & 4.2 & $3 \pm 0.5$ & $1.2 \pm 0.1$ & [9] \\
\hline
\end{tabular}

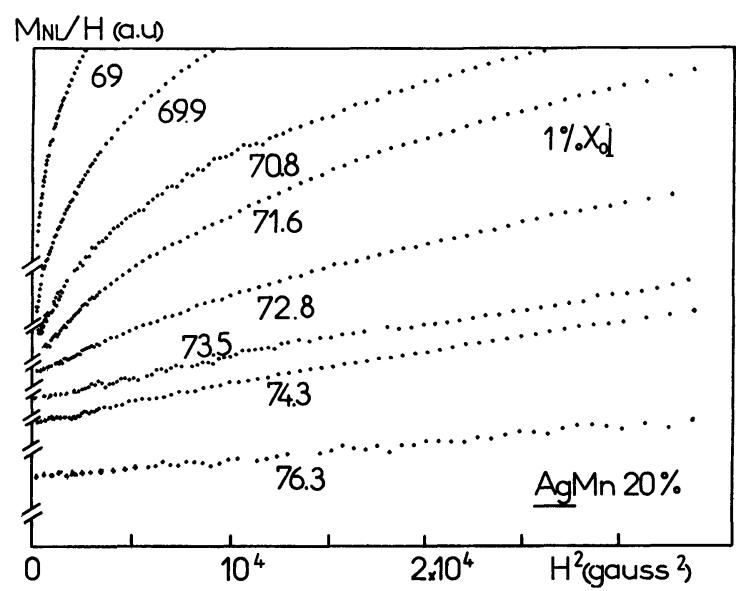

Fig. 10. - Typical set of curves yielding $\Delta M / H$ as a function of the square of the magnetic field for different temperatures above the transition temperature on the 20.5 at. \% sample. The initial slope of each curves, yields $a(T)$.

zero Curie Weiss temperature $\theta$ determine by analysing the low field magnetization as a function of temperature : $M / H=\frac{C}{T-\theta}$ in the neighbourhood of $T_{\mathrm{g}}$. For the 20 at. $\%, \theta=22 \pm 0.5 \mathrm{~K}$. Instead of $a(T)$ the quantity plotted in figure 11 is $a^{\prime}(T)=\left(1-\frac{\theta}{T}\right)^{4} a(T)$

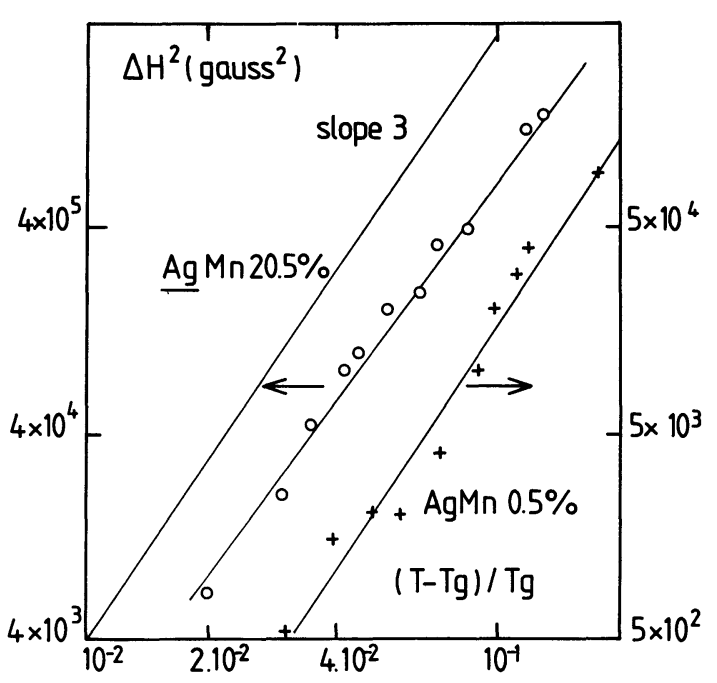

Fig. 11. - Square of the range of magnetic field used for the determination of $a(T)$ versus $\left(T-T_{\mathrm{g}}\right) / T_{\mathrm{g}}+\operatorname{AgMn} 0.5 \%$; O AgMn $20.5 \%$.

(assuming like in (9) that the existence of such a Curie Weiss behaviour modifies the scaling behaviour of the nonlinear magnetization in the following way :

$$
M /\left(H+J_{0} M\right)-\frac{C}{T}=\tau^{\beta} f\left(\frac{\left(H+J_{0} M\right)^{2}}{\tau \phi}\right)
$$


with $J_{0}=\theta / C$ ). However in the temperature range between $T_{\mathrm{g}}$ and $1.1 T_{\mathrm{g}}$ which we have considered, this correction is of minor importance and changes the value of $\gamma$ from less than $5 \%$. In the temperature range between $T_{\mathrm{g}}$ and $1.1 T_{\mathrm{g}}, a(T)$ is well described by a power law (see Fig. 12) :

$$
a(T) \propto\left(T-T_{\mathrm{g}}\right)^{-\gamma}
$$

with

$$
\begin{aligned}
& \gamma=2.1 \pm 0.1 \text { for the } 0.5 \text { at. } \% \text { sample } \\
& \gamma=2.3 \pm 0.1 \text { for the } 20.5 \text { at. } \% \text { sample }
\end{aligned}
$$

Deviations from this behaviour are observed for the 0.5 at. \% between $1.2 T_{\mathrm{g}}$ and $1.5 \mathrm{~T}_{\mathrm{g}}$ as seen in figure 11 . These deviations would yield an apparent value of $\gamma=3$ in the range of temperatures between 1.1 and $1.5 T_{\mathrm{g}}$ [27]. Are these deviations the manifestation of the presence of regular terms which are expected (See Sect. 2) to contribute subsequently to the nonlinear magnetization at temperatures above (1.1) $T_{\mathrm{g}}$ ? Or are they the manifestation of a crossover from one type of critical behaviour to another characterized by different values of critical exponents ? This point will be discussed in section 4 .

\subsection{ANAL YSIS OF THE SLOPE .IT THE INFLEXION POINT OF} $M_{\mathrm{N} J} / H$. Determination of $\beta$. - An examination of the set of $M_{\mathrm{NL}} / H=f(H)$ curves in figure 6 shows that each of these curves present an inflexion point whose position along the field axis decreases towards zero when the temperature decreases towards $T_{\mathrm{g}}$. The position of this inflexion point is not well defined but the slope $p$ of the tangent of the curve at this inflexion point can be accurately measured. It is

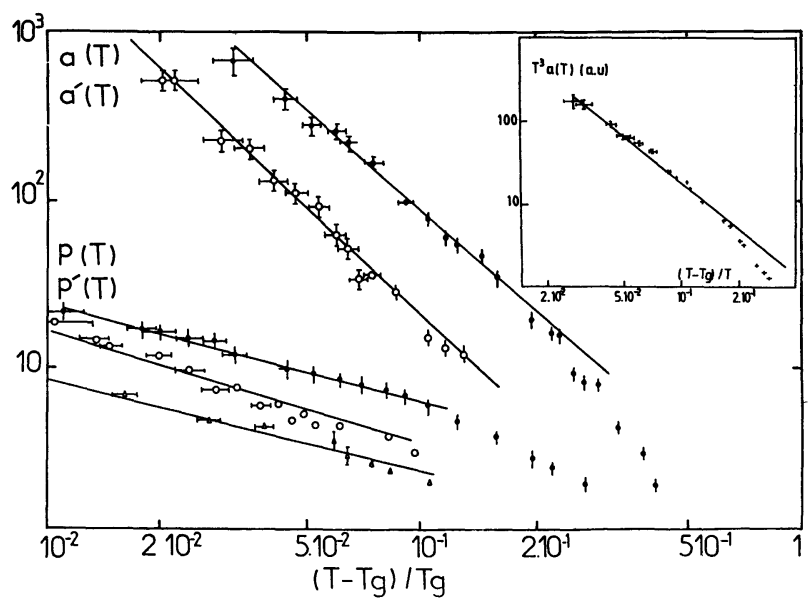

Fig. 12. - Temperature dependence of the quadratic term in field of $\Delta M / H: a(T)$ from which one reads the value of $\gamma:$ - 0.5 at. $\%$ sample; 020.5 at. \% sample : $a^{\prime}(T)=a(T)$ $(1-\theta / T)^{4}$. Temperature dependence of the slope of the tangent of $\Delta M / H=f(H)$ measured at the inflexion point : $p(T)$ from which one reads the value of $\psi=(\gamma-\beta) / 2$. - 0.5 at. $\%$ sample; $\triangle 0.7$ at. $\%$ sample; $O 20.5$ at. $\%$ sample : $p^{\prime}(T)=p(T)(1-\theta / T)^{3}$. Insert : plot of $T^{3} a(T)$ using $\left(T-T_{\mathrm{g}}\right) / T$ as scaling variable. plotted in (Fig. 11) as a function of $\left(T-T_{\mathrm{g}}\right)$, in the temperature range below $1.1 T_{\mathrm{g}}$ it can be well described by :

$$
p=\left(T-T_{\mathrm{g}}\right)^{-\psi} \text { where } \quad \psi=0.6 \pm 0.05 .
$$

Such a behaviour can be understood by assuming that the nonlinear magnetization is properly described by the scaling assumption :

$$
K_{\mathrm{NL}}=M_{\mathrm{NL}} / H=\tau^{\beta} F(x)
$$

where

$$
\begin{gathered}
x=H^{2} / \tau^{\phi} ; \quad \lim _{x \rightarrow 0} F(x)=x \text { and } \lim _{x \rightarrow \infty} F(x)=x^{1 / \delta} \\
\frac{\mathrm{d}}{\mathrm{d} H} K_{\mathrm{NL}}=\frac{2 H \tau^{\beta}}{\tau^{\phi}} F^{\prime}(x) .
\end{gathered}
$$

At the inflexion point :

$$
\begin{gathered}
\frac{\mathrm{d}^{2}}{\mathrm{~d} H^{2}} K_{\mathrm{NL}}=\frac{2 \tau^{\beta}}{\tau^{\phi}}\left(F^{\prime}(x)+\frac{2 H^{2}}{\tau^{\phi}} F^{\prime \prime}(x)\right)=0 \\
H^{2} / \tau^{\phi}=x_{0} \text { with } \quad F^{\prime}\left(x_{0}\right)+2 x_{0} F^{\prime \prime}\left(x_{0}\right)=0 .
\end{gathered}
$$

The corresponding slope $p$ verifies :

$$
\begin{gathered}
p=\left.\frac{\mathrm{d}}{\mathrm{d} H} K_{\mathrm{N}}\right|_{x=x_{0}}=2 x_{0}^{1 / 2} F^{\prime}\left(x_{0}\right) \tau^{(\beta-\gamma) / 2} \\
p \propto\left(T-T_{\mathrm{g}}\right)^{-\psi} \\
\psi=\frac{\beta-\gamma}{2}=\gamma(\delta-2) / 2(\delta-1)
\end{gathered}
$$

(using the relation : $\beta \delta=\beta+\gamma$ )

$p$ is thus temperature independent in the mean field case for which $\delta=2$. This can be seen in figure 13 . The temperature dependence of the tangent at the inflexion point can thus be used as a simple experimental criterion to distinguish an array of $K_{\mathrm{NL}}$ mean field isotherms from a non mean field one for which

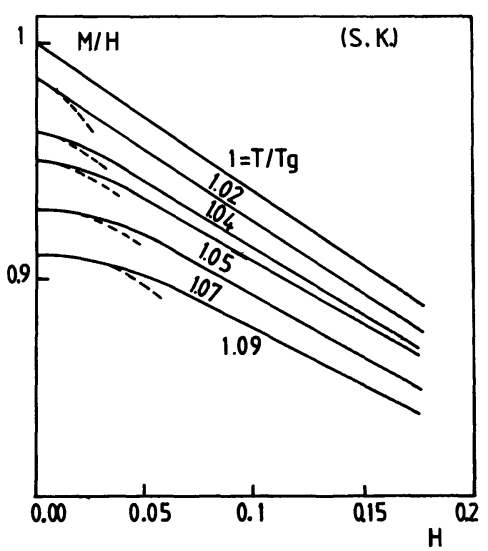

Fig. 13. - Set of curves yielding $M / H=F(H)$ calculated from the mean field S.K. solution. Note that each curve presents an inflexion point at which the slope of the tangent is temperature independent. 
$\delta>2$. Our experimental determinations of $\psi$ in the temperature range below $1.1 T_{\mathrm{g}}$ are :

\begin{tabular}{c|c|c|c}
$C$ at. \% & 0.5 & 0.7 & 20.5 \\
\hline$\psi$ & $0.6 \pm 0.05$ & $0.6 \pm 0.05$ & $0.7 \pm 0.05$
\end{tabular}

The deviations towards higher values of $\psi$ observed in $p(T)$ for $T>1.1 T_{\mathrm{g}}$ raise the same questions as the deviations noted in the study of the temperature dependence of $\chi_{\mathrm{NL}}$ in 3. From our determinations of $\gamma$ and $\psi$ we can deduce from (17) $\beta=1 \pm 0.1$ for the 0.5 and 20 at. \% samples. We can verify from the knowledge of $\delta=3.1 \pm 0.2$ determined in 3.1 and $\psi$ that relation (17) yields :

$$
\begin{array}{ll}
\gamma=2 \psi \frac{(\delta-1)}{(\delta-2)}=2.3 \pm 0.2 & \text { for the } 0.5 \\
\gamma=2.4 \pm 0.2 & \text { for the } 20.5 \text { at. } \%
\end{array}
$$

which is indeed in agreement with our previous independent determinations of $\gamma$ in 3.2. Our three independently measured critical exponents $\beta, \gamma$ and $\delta$ thus verify the scaling relation :

$$
\beta \delta=\beta+\gamma .
$$

This result a posteriori justifies our choice for the transition temperature. A shift of $T_{\mathrm{g}}$ of more than $0.5 \%$ would indeed modify $\gamma$ and $\psi$ in such a way that the scaling relation (18) would not be verified (within the $10 \%$ error bar of our determination of the critical exponents) [28].

3.4 SCAling Function [29]. - We have tried to describe our experimental data with a scaling function :

$$
K_{\mathrm{NL}}=\tau^{\beta} F\left(H^{2} / \tau^{\phi}\right)
$$

in the temperature range where $\tau<0.1$ and the range of magnetic field where $K_{\mathrm{NI}} / \chi_{0}<0.1$. The best fit is obtained for $\phi=3.3 \pm 0.1$ and $\beta=1 \pm 0.1$ independent of the Mn concentration see figure 14 for the AgMn 0.5, 0.7 and 20.5 at. \% samples [30]. The exponent $\phi$ verifies the scaling relation $\gamma+\beta=\phi$.

Indeed, our previous determination of $\gamma$ yields :

and

$$
\gamma+\beta=3.1 \pm 0.2 \text { for the } 0.5 \text { at. } \%
$$

$$
\gamma+\beta=3.3 \pm 0.2 \text { for the } 20 \text { at. } \% \text {. }
$$

The asymptotic forms of the scaling function of the $x=H^{2} / \tau^{\phi}$ variable can also be checked in figure 14 :

1) $F(x)$ is linear for small $x$ which corresponds to the range of fields and temperatures where $K_{\mathrm{NL}}$ is dominated by the quadratic term (i.e. $H<\Delta H$ defined in 3.2). One can indeed verify in figure 11 that $\Delta H^{2}$ scales like $\tau^{3} \sim \tau^{\phi}$.

2) $F(x)$ varies like $x^{1 / \delta}$ for high values of $x$. It is note worthy to find the same values for the critical exponents in the dilute systems $(0.4,0.5,0.7)$ at. $\%$ and in the concentrated system (20.5 at. \%). In the latter case indeed, the respective number of first and second neighbours deduced from short range order parameters determined by $\mathrm{X}$ ray scattering studies on the same sample [31] are $N_{1}=1$ and $N_{2}=2.3$. First and second neighbour magnetic interactions are thus expected to play the main role at the spin glass transition. The AgMn $20 \%$ system may then be considered as a short range spin glass.

On the other hand in the dilute regime (i.e. $c<1 \%$ ), the magnetic interactions responsible for the transition are expected to be well described by the asymptotic $1 / R^{3}$ form of the Ruderman Kittel interaction.

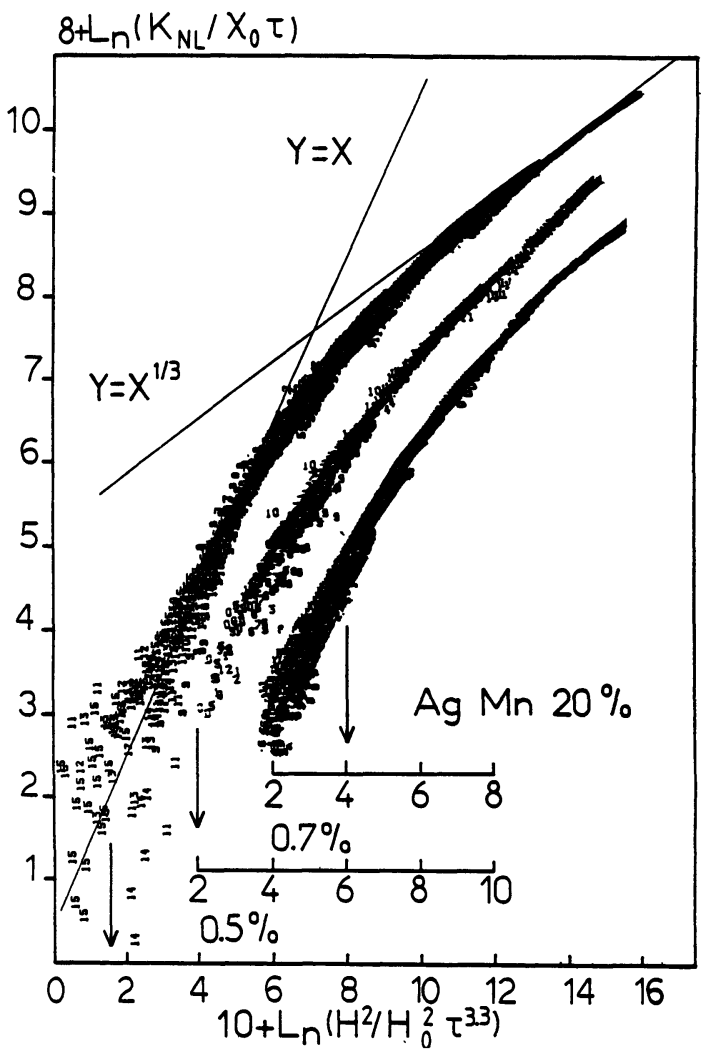

Fig. 14. - Scaling of the nonlinear magnetization expressed in units of $\chi_{0}$, as a function of the reduced field $H / H_{0}$ (where $H_{0}$ is given in table I) and the reduced temperature $\left(T-T_{\mathrm{g}}\right) /$ $T_{\mathbf{g}}$ :

18 values of $T$ and 200 values of $H$

$$
\left.\begin{array}{rl}
2.73 & <T<3.1 \mathrm{~K} \\
0 & <H<400 \text { gauss }
\end{array}\right\} 0.5 \text { at. } \%
$$

11 values of $T$ and 100 values of $H$

$$
\left.\begin{array}{rl}
3.84 & <T<4.20 \mathrm{~K} \\
0 & <H<300 \text { gauss }
\end{array}\right\} 0.7 \text { at. } \%
$$

12 values of $T$ and 200 values of $H$

$$
\left.\begin{array}{rl}
69 \mathrm{~K} & <T<77 \mathrm{~K} \\
0 & <H<1500 \text { gauss }
\end{array}\right\} 20.5 \text { at. } \%
$$

Note the shifted origins on the $x$ axis for the three different concentrations. The three scaling curves are nearly identical. 
Our result, that the critical exponents are concentration independent in AgMn is not in contradiction with the statement [32] that the R.K.K.Y. interaction has to be considered as short range from the phase transition point of view.

3.5 SCALING BeLow $T_{\mathrm{g}}$ - Nonlinear magnetization measurements have also been performed on the AgMn $0.5 \%$ sample below the transition temperature. The magnetization becomes an irreversible function of the magnetic field : the quantity $\Delta M / H$ is depicted in figure 15 at a temperature $T_{\mathrm{g}}-\Delta T$, the sample having been cooled in zero field till $T=T_{\mathrm{g}}-\Delta T$. $\Delta M / H$ measured when first increasing the magnetic field is lower than $\Delta M / H$ measured when subsequently decreasing the magnetic field for values of the magnetic field $H<H_{\mathrm{ir}}$. For $H>H_{\mathrm{ir}}$ the magnetization is again observed to be reversible. The nonlinear magnetization in this regime is equal to the nonlinear magnetization measured at $T=T_{\mathrm{g}}+\Delta T$. This fact suggests that we can check the validity of the scaling below $T_{\mathrm{g}}$ in the range of field and temperature where the magnetization is a reversible function of the magnetic field. Below $T_{\mathrm{g}}$ one expects that the linear part of the magnetization also contains a contribution of the spin glass order parameter in zero field which varies like :

$$
q=a\left(T_{\mathrm{g}}-T\right)^{\beta} \quad \text { in zero field }
$$

We thus consider the quantity :

$$
K_{\mathrm{s}}(T, H)=C / T-M / H
$$

where $C / T$ is the extrapolation below $T_{\mathrm{g}}$ of the Curie law which describes well the temperature dependence of the linear part of the magnetization above $T_{\mathrm{g}}$. $K_{\mathrm{s}}(T, H) /\left(T-T_{\mathrm{g}}\right)$ is plotted in figure 16 versus $x=H^{2} /\left(T_{\mathrm{g}} / T\right)^{3.3^{8}}$ in the range of magnetic field

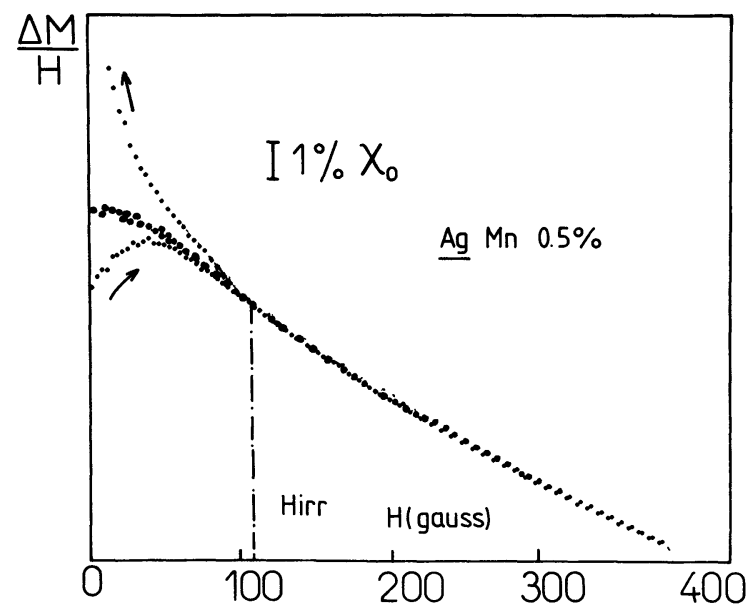

Fig. 15. - Nonlinear magnetization (small points) measured at $2.5 \mathrm{~K}=T_{\mathrm{g}}-0.2 \mathrm{~K}$ on the 0.5 at. $\%$ sample after zero field cooling compared to the non linear magnetization measured at $T=2.9 \mathrm{~K}=T_{\mathrm{g}}+0.2 \mathrm{~K}$.

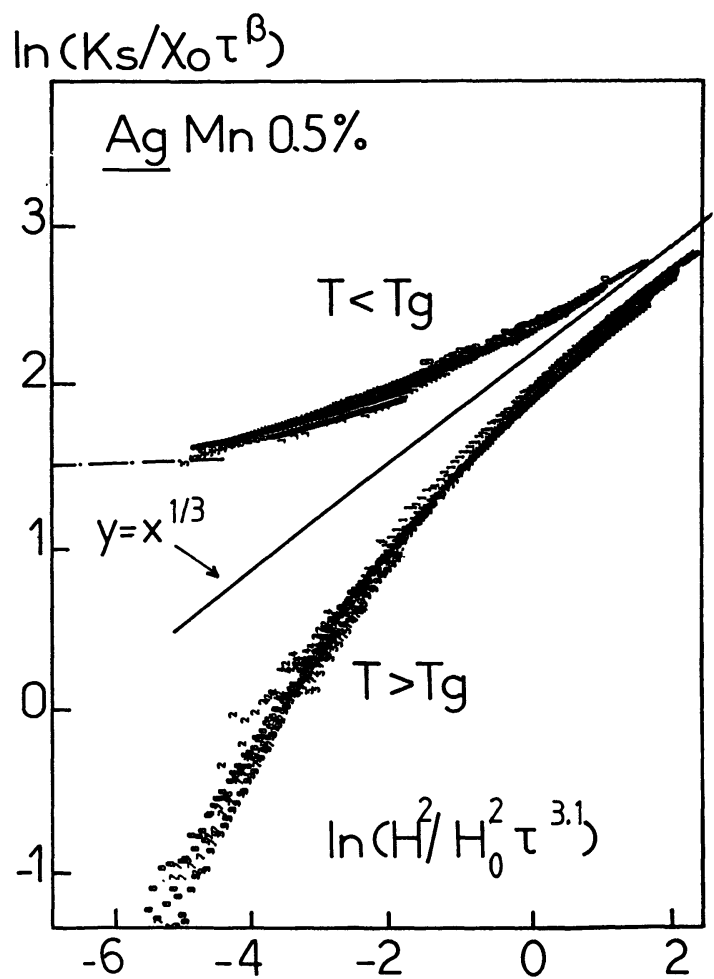

Fig. 16. - Scaling function of AgMn $0.5 \%$ for 10 temperatures above and 10 temperatures below $T_{\mathrm{g}}$ verifying $\left|T-T_{\mathrm{g}}\right| / T_{\mathrm{g}}<0.1$. Below $T_{\mathrm{g}}$ our analysis was restricted to the range of field where $H<H_{\mathrm{irr}}(T)$.

$H_{\mathrm{ir}}(T)<H<600$ gauss, for ten different temperatures below $T_{\mathbf{g}}$. The experimental points are superimposed on a universal scaling function of the variable $x$. The asymptotic behaviour for large values of $x$ :

$$
\lim _{x \rightarrow \infty} F^{\prime}(x)=x^{1 / 3}
$$

is identical to the asymptotic behaviour of the scaling function above $T_{\mathrm{g}}$. The asymptotic behaviour for small values of $x$ is :

$$
\lim _{x \rightarrow 0} F^{\prime}(x)=\text { Cte }
$$

which means that the quantity $K_{\mathrm{s}}$ is dominated by the $a\left(T_{\mathrm{g}}-T\right)$ contribution of the order parameter in zero field.

This last point can be checked in another independent way by considering below $T_{\mathrm{g}}$ the variation with temperature of the quantity $Q(T)=T(M(T)-$ $C / T)$ where $M(T)$ is the field cooled magnetization measured in low magnetic field (see Fig. 17). $q(T)$ is found to vary linearly with temperatures between $T_{\mathrm{g}}$ and $0.8 T_{\mathrm{g}}$ in agreement with $\beta=1$. The nonzero value of $q(T)$ at $T=T_{\mathrm{g}}$ is due to the finite value of the magnetic field and is in agreement with the expected contribution :

$$
q\left(T_{\mathrm{g}}\right)=\chi_{0} T_{\mathrm{g}}\left(H / H_{0}\right)^{2 / \delta}=0.22 \times 10^{-5} \text { c.g.s. } .
$$




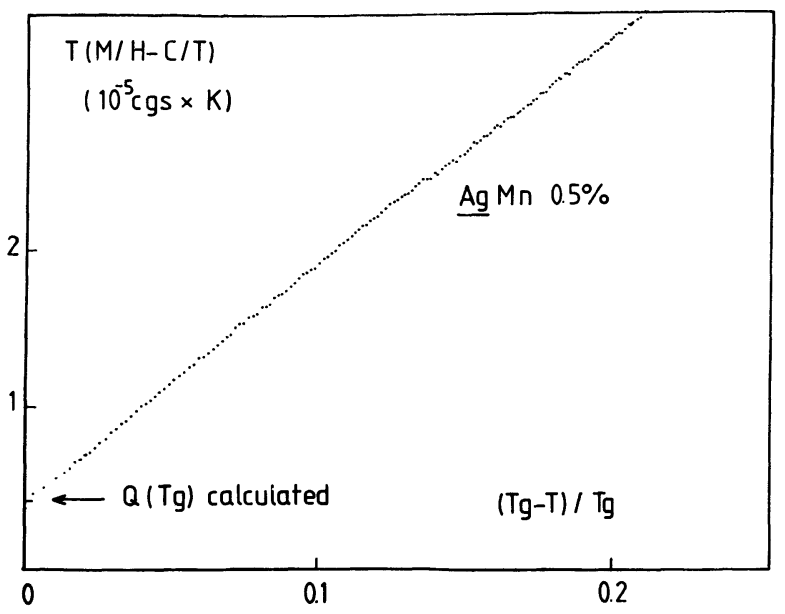

Fig. 17. - Temperature dependence of the spin glass order parameter below $T_{\mathbf{g}}$ extracted from the field cooled magnetization of $\mathrm{AgMn} 0.5 \%$ under the field $H=43$ gauss (see Fig. 18).

Note the surprisingly large temperature range of validity of the formula :

$$
q=q\left(T_{\mathrm{g}}\right)+a\left(T_{\mathrm{g}}-T\right)
$$

which is expected to be valid only in a temperature range where irreversibilities can be neglected.

3.6 ANALYSIS OF THE Magnetization as a FUNCTION OF THE TEMPERATURE. PROPERTIES RELATED TO CRITICAL EXPONENTS. - In principle the analysis of the nonlinear magnetization could be performed using a set of $M(T)$ curves for different values of the magnetic field instead of the array of isothermal curves we have considered above. The subtraction of the linear part of the magnetization is not so straightforward in the analysis of $M(T)$ curves as in $M(H)$ curves, however information on the critical exponents can be deduced. In the following we discuss two properties of the field dependence of the $M(T)$ curves which yield information on the critical exponents from the S.Q.U.I.D. measurements on AgMn of Chamberlin et al. [33]. 3.6.1 Inflexion point of $M(T)$. - In the frame-work of the scaling analysis of the nonlinear magnetization above $T_{\mathrm{g}}$ one has :

$$
\begin{gathered}
M(T)=\frac{H}{T}-a \frac{H^{3}}{\tau^{\gamma}}\left(1+0\left(H^{2} / \tau^{\gamma+\beta}\right)\right) \\
\frac{1}{H} \frac{\mathrm{d}^{2} M}{\mathrm{~d} T^{2}}=\frac{2}{T^{3}}-a \frac{H^{2}}{\tau^{\gamma+2}}\left(1+0\left(H^{2} / \tau^{\gamma+\beta}\right)\right) .
\end{gathered}
$$

The inflexion point of $M(T)$ verifies :

$$
H_{i}^{2} \propto \tau_{i}^{\gamma+2} .
$$

(Since $\gamma+2>\gamma+\beta$, the relation $H_{\mathrm{i}}^{2} \ll \tau_{\mathrm{i}}^{\phi}$ is verified and thus justifies keeping only the leading term in $\mathrm{H}^{3}$ in the nonlinear magnetization.) This criterion has already been used by Berton et al. [4] for the determination of $\gamma$ by magnetocaloric effect in CuMn. On the other hand, Chamberlin et al. [33] in their D.C. magnetization work on AgMn $2.6 \%$ and $\mathrm{AgMn} 4 \%$, have studied the inflexion point of $M(T)$ as a function of the magnetic field in the low field regime. They find

$$
T_{\mathrm{i}}-T_{\mathrm{g}} \propto H^{b}
$$

where $b=0.51 \pm 0.1$ and $0.56 \pm 0.1$ for the 2.6 at. \% and 4.0 at. $\%$ samples. They interpret this $T_{\mathrm{i}}(H)$ line as a cross-over line without trying to relate the exponent $b$ to the critical exponents of the spin glass transition. This can be done using relation (21) and one obtains :

$\gamma=2 / 0.51-2=1.9 \pm 0.3$ for the 2.6 at. $\%$ sample and

$\gamma=2 / 0.56-2=1.8 \pm 0.3$ for the 4 at. $\%$ sample.

These values are in agreement with our own determination of $\gamma$ on AgMn using a completely independent criterion. One can note that like our own analysis, the analysis of Chamberlin et al. is restricted to the range of temperatures and fields where $\left(T-T_{\mathbf{g}}\right) /$ $T_{\mathrm{g}}<10^{-1}$ and $M_{\mathrm{NL}} / M<10^{-1}$.

3.6.2 Maximum of $M(T)$. - In [34] we have shown that the field dependence of the maximum of the magnetization can be related to the critical exponents of the spin glass transition.

When $\beta<1$ this maximum is found to coincide with $T_{\mathrm{g}}$ in the limit of zero magnetic field and to move up in temperature with increasing field.

When $\beta>1$, the maximum of $M(T)$ is predicted at a temperature lower than $T_{\mathbf{g}}$ where the magnetization becomes irreversible.

In our $0.5 \%$ AgMn sample (see Fig. 4 and Fig. 18) the low field limit of the maximum of $M(T)$ coincides (within better than $0.5 \%$ ) with the occurrence of irreversibility and moves down in temperature with increasing magnetic field. This behaviour can only be understood if $\beta=1$ (the mean field value), which indeed confirms our previous determination. This experimental fact has already been observed by Chamberlin et al. [33] for the 2.6 and 4 at. \% systems.

Furthermore the low field dependence of the maximum of $M(T)$ is found to follow an $H^{2 / 3}$ law approximately. Such a behaviour is in agreement with the $H^{2 / \gamma+\beta}$ power law expected in that case [34], the critical exponents $\gamma$ and $\beta$ being determined from our nonlinear magnetization data.

Although these analyses are less precise and more qualitative, they yield complementary and independent information which confirms our determination of the critical exponents from the analysis of magnetization isotherms.

3.7 Evaluation OF THE OTHER CRITICAL EXPONENTS. - From the knowledge of the exponents $\beta, \gamma$ and $\delta$, other critical exponents can be calculated from the scaling relations. 


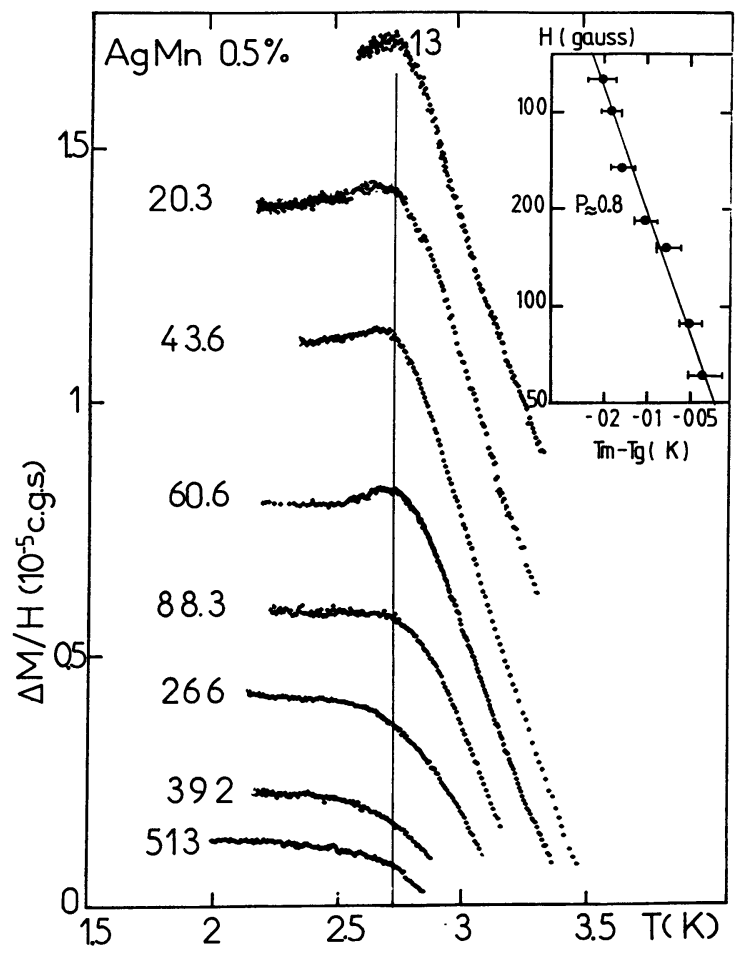

Fig. 18. - Set of curves yielding the temperature dependence of the field cooled magnetization for the 0.5 at. $\%$ sample at various values of the magnetic fields, the origins on the $Y$ axis have been arbitrarily shifted. Insert : field dependence of the maximum of the field cooled magnetization.

3.7.1.- Exponent of the specific heat.-C(T) $\alpha\left(T-T_{\mathrm{g}}\right)^{-\alpha}$; it can be deduced from the relation :

$$
\begin{aligned}
& \alpha+2 \beta+\gamma=2 \\
& \alpha=-2.2 \pm 0.2
\end{aligned}
$$

This result yields a specific heat anomaly of its third temperature derivative and is thus in agreement with the absence of anomaly observed experimentally [35].

3.7.2 Exponents concerning the correlation length $\xi(T)$ : the scaling behaviour of the spatial correlation function of the spin glass order parameter is expected to be in the vicinity of $T_{\mathrm{g}}$ :

$$
\begin{aligned}
G(R)=\frac{1}{V} \sum_{R^{\prime}}\left\langle S(\mathbf{R}) S\left(\mathbf{R}+\mathbf{R}^{\prime}\right)\right\rangle^{2} & \\
= & \frac{1}{R^{d-2+\eta}} \mathrm{e}^{-R / \xi(T)}
\end{aligned}
$$

with

$$
\xi(T) \propto\left(T-T_{\mathrm{g}}\right)^{-v}
$$

$\eta$ and $v$ can be deduced from the relations :

$$
\begin{gathered}
d v=2-\alpha \quad \text { which yields } \quad v=1.3 \pm 0.1 \\
2-\eta=\gamma / v \quad \eta=0.3 \pm 0.1 .
\end{gathered}
$$

These values can be compared to the values $v=1.2$ and $\eta=0$ obtained by Ogielski et al. [16] in their numerical simulations of a 3-dimensional Ising spin glass.

3.8 COMPARISON OF CRITICAL EXPONENTS DETERMINED BY D.C. MEASUREMENTS AND BY A.C. MEASUREMENTS. A.C. susceptibility measurements, with a D.C. applied field varying between 1 and 800 gauss superimposed on the A.C. exciting field $(f=32 \mathrm{~Hz})$, were also done on the 0.7 at. \% sample in collaboration with Beauvillain and Renard on the same set up they used for their study of $\mathrm{Al}_{2} \mathrm{O}_{3} \mathrm{MnOSiO}_{2}$ [7]. In the absence of frequency dependence effects, one expects that the A.C. susceptibility depends on the D.C. applied field $H$ like :

$$
\begin{gathered}
T>T_{\mathrm{g}}: \chi_{\mathrm{AC}}=\frac{\mathrm{d} M}{\mathrm{~d} H}=\chi(T)-3 a(T) H^{2}+0\left(H^{4}\right) \\
T=T_{\mathrm{g}}: \chi_{\mathrm{AC}}=\chi_{0}\left(1-(1+2 / \delta)\left(\frac{H}{H_{0}}\right)^{2 / \delta}\right)
\end{gathered}
$$

with the notations of 3.1 and 3.2 .

The amplitude of the $H^{2}$ term of $\mathrm{d} M / \mathrm{d} H: \tilde{a}(T)$ is well described between $T_{\mathrm{g}}$ and $1.1 T_{\mathrm{g}}$ by : $\tilde{a}(T) \propto$ $\left(T-T_{\mathrm{g}}\right)^{-\gamma}$ with $\tilde{\gamma}=1.8 \pm 0.1$ (see figure 19) which is a little lower than the value $\gamma=2.2$ obtained by our D.C. analysis. The nonlinear part of the susceptibility at $T_{\mathrm{g}}$ plotted on a double logarithmic scale presents

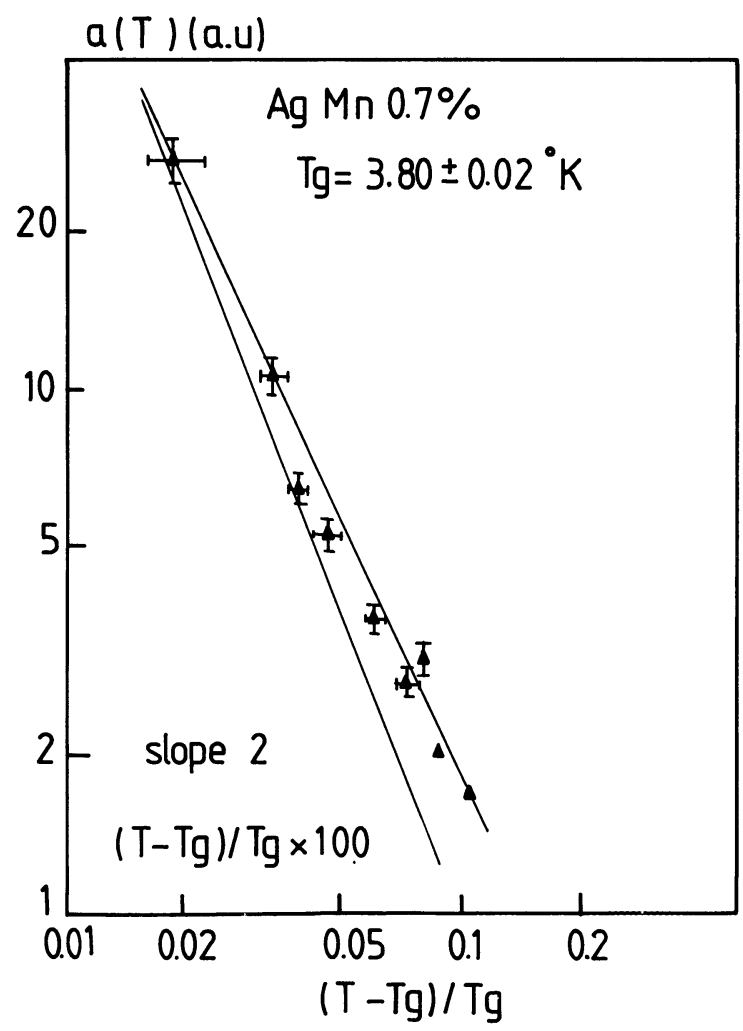

Fig. 19. - Temperature dependence of the quadratic term in field of $\mathrm{d} M / \mathrm{d} H$ measured by A.C. susceptibility on AgMn $0.7 \%$ between $T_{\mathrm{g}}$ and $1.1 T_{\mathrm{g}}$. 


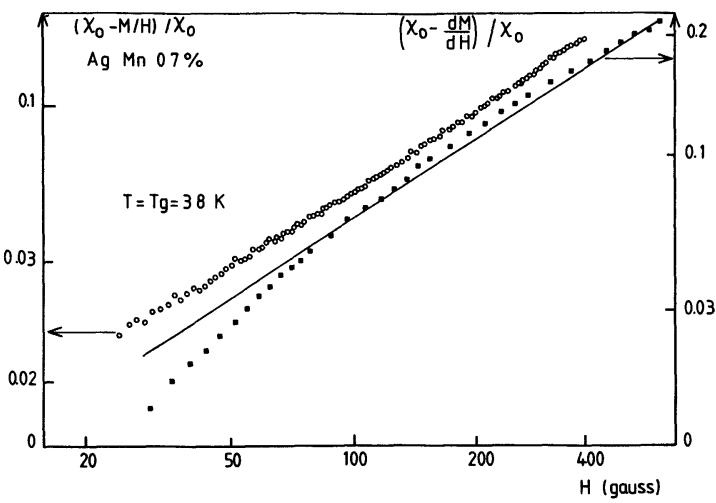

Fig. 20. - Field dependence of $\mathrm{d} M / \mathrm{d} H$ measured by A.C. susceptibility at $T=T_{\mathrm{g}}$ (note the curvature observed in the low field range), compared to the non linear magnetization measured by our D.C. technique.

a slight curvature (see figure 20) in low field. Its analysis between 100 and 800 gauss yields $\delta=3 \pm 0.2$ compatible with our D.C. results. An analysis in the range of fields below 100 gauss would yield a value of $\delta \approx 2$. This result should be compared to the result of Mulder et al. on CuMn [36] whose similar analysis of the field dependence of $\chi_{\mathrm{AC}}$ at $T=T_{\mathrm{g}}$ yields $\delta=2$ in the range of magnetic fields where $\chi_{\mathrm{NL}} / \chi_{0}<2 \times 10^{-2}$. We attribute this disagreement between the A.C. and D.C. determinations of $\delta$ in low magnetic fields to frequency dependent effects.

\section{Discussion of the results.}

4.1 CAN WE ELIMINATE THE POSSIBILITY OF A ZERO TEMPERATURE TRANSITION ? - In the preceding section we have analysed our results within the framework of the existence of a phase transition at $T_{\mathbf{g}}$. We have tried to extract the critical exponents from our data in as many as possible independent ways according to this finite temperature phase transition hypothesis. As far as we have done we have not met with any contradiction. In the following we compare our results to some of the predictions of the $T_{\mathrm{g}}=0$ scaling hypothesis. Binder and Kinzel have analysed their numerical results of the bidimensional Ising spin glass model in such a framework and suggested extending it to the analysis of experimental data as well [11 to 13].

4.1.1 Temperature dependence of the $\mathrm{H}^{3}$ term of the nonlinear magnetization. - The coefficient of the quadratic term of $M_{\mathrm{NL}} / H=a(T)$ is plotted against temperature on a double logarithmic scale in figure 21. One can make two points :

1) this plot appears to be nonlinear even when the temperature varies by a factor smaller than 1.3.

2) The quantity $\frac{\mathrm{d} \log a}{\mathrm{~d} \log T}$ varies from 15 to 30 in the same temperature range. The $T=0$ scaling hypothesis prediction $a(T)=T^{-\gamma *}$ would thus yield unphysical values for the critical exponent $\left(\gamma^{*}>30\right)$.

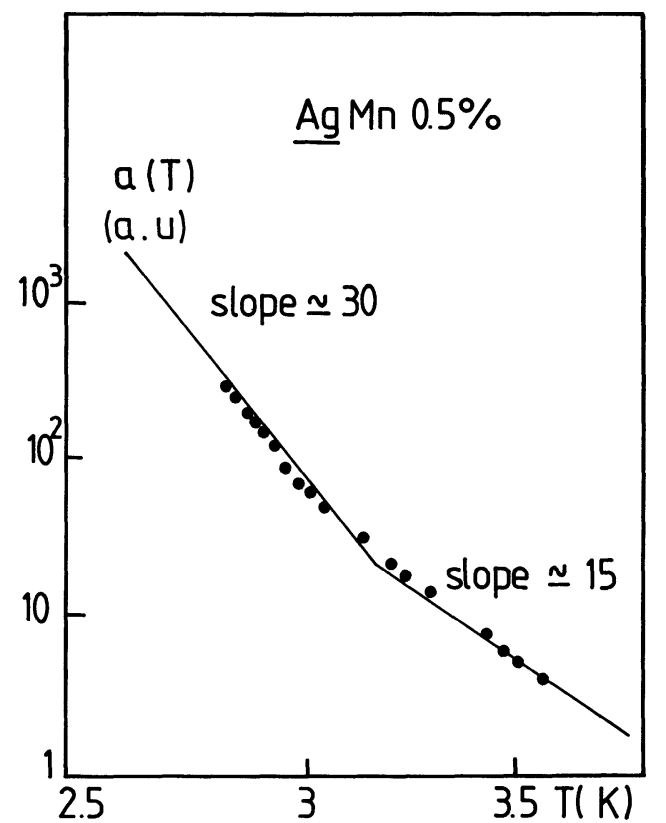

Fig. 21. - Non linear susceptibility as a function of temperature between $T_{\mathrm{g}}$ and $1.3 T_{\mathrm{g}}$ on a double logarithmic scale, the value of $\gamma$ deduced from the $T_{\mathrm{c}}=0$ scaling hypothesis is higher than 30 .

In figure $22 a(T)$ is plotted on a semilogarithmic scale. The curvature observed shows that $a(T)$ cannot be described by :

$$
a(T) \propto \exp \alpha T_{\mathrm{g}} / T
$$

as expected at the lower critical dimension [37]. In our case a possible fit for $a(T)$ could be $a(T)=\mathrm{e}^{\alpha\left(T / T_{\mathrm{g}}\right)^{\sigma}}$ with $\sigma=3.1 \pm 0.4$ and $\alpha \approx 10$ which again is unphysical. We therefore conclude that our observation of a three order of magnitude increase of $a(T)$ over a small temperature range (between 1.02 and $1.3 T_{\mathrm{g}}$ ) is ruling out any reasonable analysis with a zero transition temperature.

4.1.2 Field dependence of the magnetization. - Another prediction of the scaling at $T=0$ is that at low temperature and in the low field regime the magnetization is expected to vary like :

$$
M / H \propto H^{-x}
$$

where $x \approx 0.3$ in the bidimensional spin glass Ising model [12]. Figure 23 shows $M / H$ versus $H$ plotted on a double logarithmic scale. The strong curvature observed, in a region of field where the nonlinear magnetization is well described by $a-H^{2 / 3}$ law (see 3.1 ), shows that (28) is completely inadequate to describe the magnetization, contrary to the statement of Binder and Kinzel in [12] who tried to fit our data of reference [3] on AgMn $10.6 \%$ with expression (28) on a limited range of fields.

4.2 COMPARISON WITH OTHER SYSTEMS. - In table II we present a list of the experimental determinations of the critical exponents of the spin glass transition. 


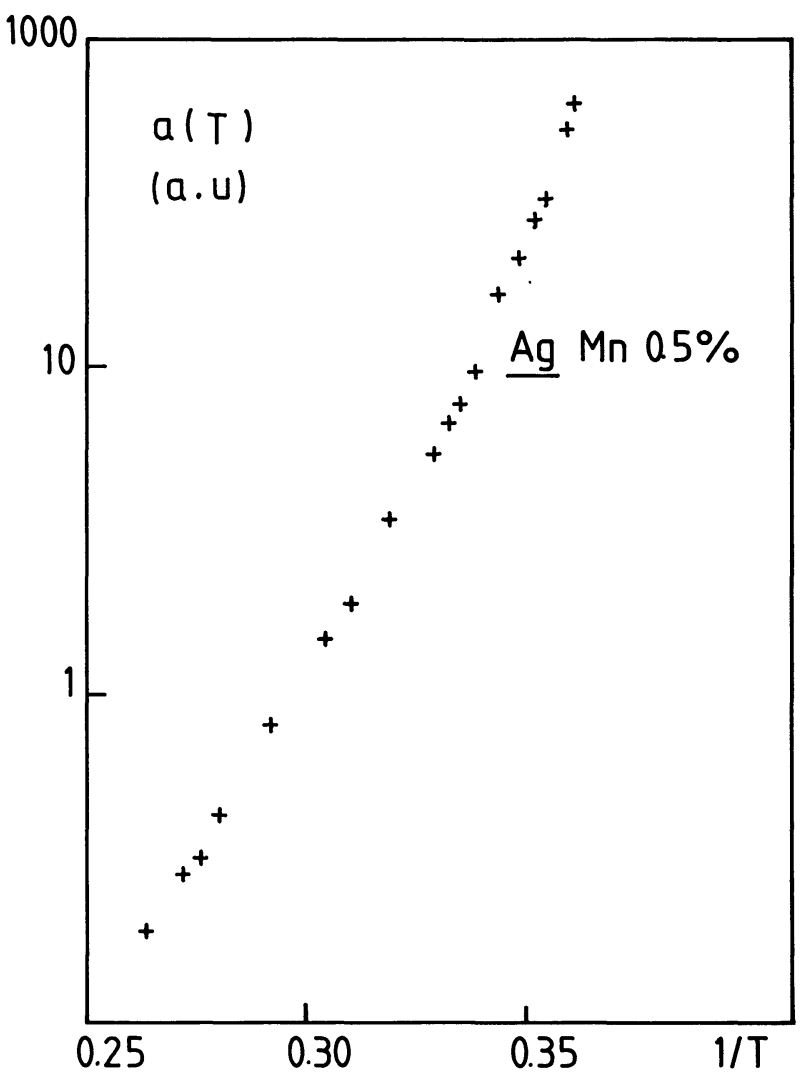

Fig. 22. - Analysis of the non linear susceptibility in the hypothesis of the lower critical dimension scaling. $\log (a(T))$ function of $1 / T$.

The wide spectrum of the values of these socalled " universal exponents " could be taken in itself as an argument against the phase transition. One however has to take into account :

- The diversity of the systems, either crystalline or amorphous, insulating or metallic, and accordingly the diversity of the techniques used, either D.C. or A.C.

- Most seriously, the diversity of the range of temperature and magnetic field studied.

The influence of the spatial disorder of the magnetic atoms on the critical behaviour has already been pointed out in the study of amorphous ferromagnets [40]. The critical exponents were found to be strongly dependent on the temperature range studied. The universal value of $\gamma$ was found only in the very vicinity of $T_{\mathrm{c}}$. The analysis of the data outside of the very vicinity of $T_{\mathrm{g}}$ yields values of $\gamma$ up to $50 \%$ above the universal value. Fähnle et al. [41] suggest that a similar behaviour should be expected in spin glasses yielding strong temperature and system dependences on the exponent of the divergence of the nonlinear susceptibility.

In some cases (see Table II) the critical exponents were determined from A.C. susceptibility measurements (analysis of the response at $3 \omega$ or of the variation of $\frac{\mathrm{d} M}{\mathrm{~d} H}$ as a function of an applied D.C. magnetic

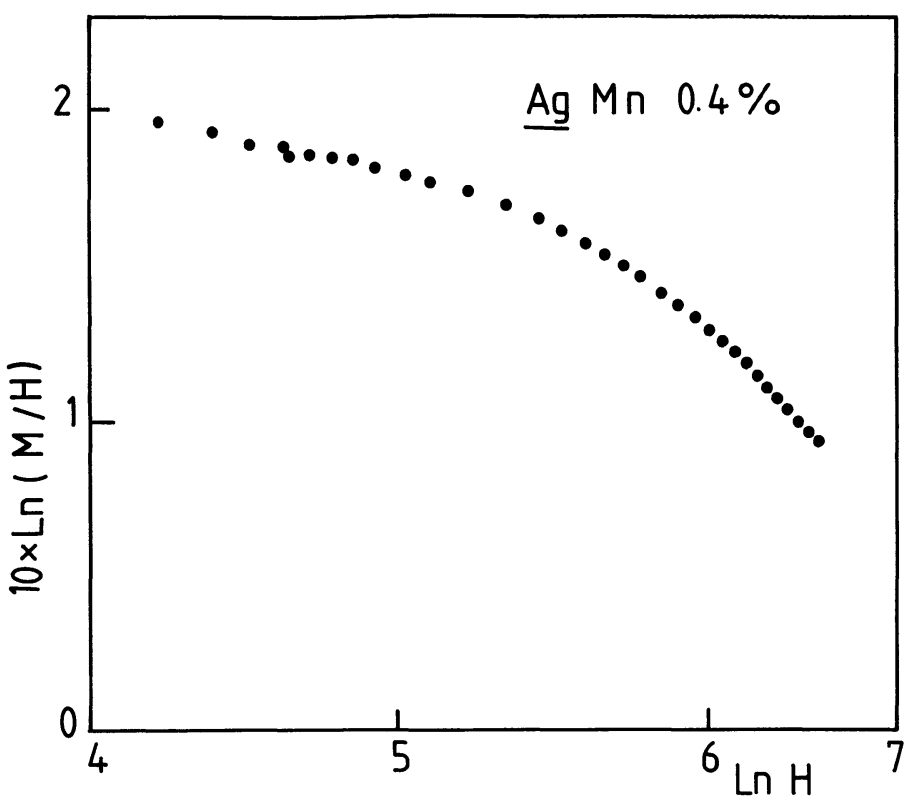

Fig. 23. $-M / H$ at $T \approx \bar{T}_{\mathrm{g}}$ for the 0.4 at. $\%$ sample is plotted versus magnetic field on a double logarithmic scale; note the strong curvature observed which excludes any reasonable fit $M / H \propto H^{-x}$.

field). One can note that the values of $\gamma$ and $\delta$ obtained with these techniques are generally lower and closer to the mean field ones than the values determined from D.C. magnetization measurements. The most striking example is the value of $\delta$ in Cu-Mn which is found equal to 5 in [5] and 2 in [35]. One may argue that frequency dependences in the vicinity of $T_{\mathrm{g}}$ can alter the analysis. This is the reason why Beauvillain $e t$ al. restricted their A.C. analysis on $\mathrm{Al}_{2} \mathrm{O}_{3} \mathrm{MnOSiO}_{2}$ (at $69 \mathrm{~Hz}$ ) in a range of temperature above $1.1 T_{\mathrm{g}}$ where frequency effects are negligible. Our own A.C. analysis on AgMn $0.7 \%$ however yields critical exponents which are compatible within $10 \%$ with the values determined by D.C. magnetization measurements in the same range of magnetic fields and temperatures (see 3.6). The problem of frequency dependences can thus not explain the observed disagreement in $\mathrm{CuMn}$ between the different estimations of $\delta$. A.C. susceptibility measurements are generally made in the very neighbourhood of $T_{\mathrm{g}}$ and in low applied D.C. magnetic fields, in contrast with D.C. measurements where the analysis was made in a wide range of magnetic field and temperature.

In order to emphasize this point we have plotted in figure 24 the value of $\delta$ versus the range of field used for its determination, expressed in terms of $M_{\mathrm{NL}} \max / M . \delta$ appears as an increasing function of $M_{\mathrm{NL}} / M$ varying between 2 and 5 [49]. We have indeed shown in our analysis on AgMn that the values of $\gamma=2$ and $\delta=3$ could describe properly the critical behaviour on the nonlinear magnetization, as far as the temperature and field range analysis where restricted to $\frac{T-T_{\mathrm{g}}}{T_{\mathrm{g}}}<0.1$ 


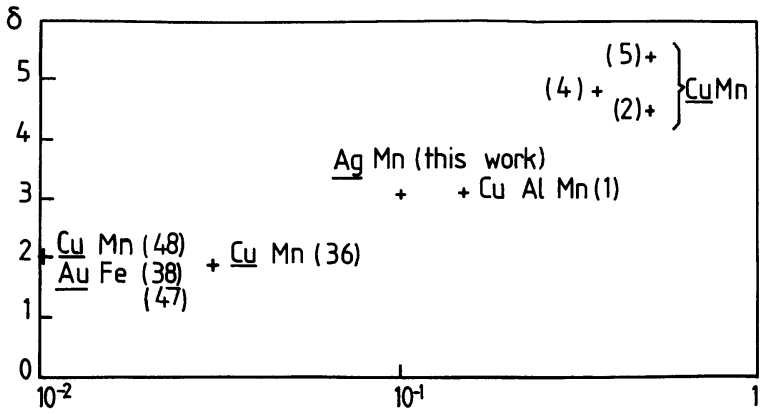

MNL / M

Fig. 24. - Measured values of the critical exponent $\delta$ on metallic spin glasses as a function of the range of magnetic field used for its determination (expressed in $M_{\mathrm{NL}} \max / M$ ).

and $M_{\mathrm{NL}} / M<0.1$. Taking data outside of that range would have yielded higher exponents $: \gamma \simeq 3, \delta \approx 5$. We think that this remark explains the disagreement between our results on AgMn and the results of Omari et al. on CuMn since these systems are otherwise expected to behave in very similar ways. We have shown in Section 1 how the presence of regular terms in the development of $M_{\mathrm{NL}}(H, T)$ could alter the determination of critical exponents for $\left(T-T_{\mathrm{g}}\right) /$ $T_{\mathrm{g}}>0.1$ and $H>0.1$, yielding $30 \%$ of error. However up to now it is not possible to evaluate the order of magnitude of the regular terms in the R.K.K.Y. metallic spin glasses. Thus the possibility of a real physical crossover occurring at $\left(T-T_{\mathrm{g}}\right) / T_{\mathrm{g}}>0.1$ can not be excluded.

The torque experiments performed by $\mathrm{N}$. de Courtenay et al. [42] on CuMn and AgMn have shown the existence of a crossover between two different regimes below $T_{\mathrm{g}}$ at $\left(T_{\mathrm{g}}-T\right) / T_{\mathrm{g}} \simeq 0.1$ in their determination of the irreversibility line. This crossover is shifted towards higher fields and lower temperatures by adding gold impurities which increase the anisotropic character [43] of the interactions. It is thus suggested that this crossover is indeed a crossover between an Ising type of behaviour (in low field near $T_{\mathrm{g}}$ ) towards a Heisenberg type of behaviour in higher magnetic field at lower temperature.

If one believes that the equation of the irreversibility line is related to the values of the critical exponents (as has been recently suggested by Fisher and Sompolinsky [44]) then one has to expect the same kind of crossover between different values of critical exponents to be observed on the nonlinear magnetization as well [45].

The values of $\gamma$ and $\delta$ that we determine in AgMn below 1.1 $T_{\mathrm{g}}$ and low field would then be characteristic of an Ising critical behaviour, as the values determined at higher temperatures and magnetic fields would be characteristic of a Heisenberg type of behaviour. This conjecture has to be confirmed by further work including the comparison of the nonlinear magnetization of CuMnAu alloys for different gold concentrations [46].
In conclusion we have shown that the nonlinear magnetization of the AgMn system can be consistently described by scaling theory with a nonzero transition temperature. The values of critical exponents $\gamma=2.2 \pm$ 0.2 and $\delta=3.1 \pm 0.2=1 \pm 0.1$ are obtained when the analysis is restricted to the range of temperatures and fields $\left(T-T_{\mathrm{g}}\right) / T_{\mathrm{g}}<0.1$ and $M_{\mathrm{NL}} / M<0.1$.

Deviations towards higher apparent values of the critical exponents are observed outside this range. These deviations explain the discrepancy between the values of the critical exponents determined by Omari et al. on CuMn and ours. Such deviations due to the presence of regular terms are also present in the mean field equation of state. However further work is needed for the physical understanding of an eventual crossover between two different kinds of critical behaviour.

\section{Acknowledgments.}

I thank P. Monod for all the stimulative help and suggestions he has given to this work. I thank P. Beauvillain and J. P. Renard for the A.C. measurements and their suggestions concerning the determination of the critical exponents. E. Brezin, T. Garel, K. Pappa, J. J. Préjean, J. Souletie and J. L. Tholence are acknowledged for stimulating discussions. T. Lumberger is acknowledged for his critical reading of the manuscript.

\section{Appendix.}

One can note the difference existing between the mean field equation of the spin glass transition and the mean field equation of the classical ferromagnetic transition. In the latter case, the development of $M(H, T)$ is exactly given by :

$$
M(H, T)=a \frac{h^{\prime}}{\tau^{\prime}}+b \frac{h^{\prime 3}}{\tau^{\prime 4}}+C(T) \frac{h^{\prime 5}}{\tau^{\prime 7}}+\cdots
$$

where $a$ and $b$ are independent of temperature $(a=1$ and $b=-1 / 3)$. This justifies, in this case, the choice of $h^{\prime}$ and $\tau^{\prime}$ as scaling variables. In this problem the magnetization is indeed the order parameter while in the spin glass problem the relation between the magnetization and the spin glass order parameter is not so direct.

Simple symmetry arguments show that the temperature and field dependence of the spin glass order parameter occur through $\mathrm{H}^{2}$ and $T^{2}$, and can be developed as follows :

$$
\begin{aligned}
q=\frac{H^{2}}{T^{2}} \frac{1}{\tau^{\prime \prime}}+\frac{H^{4}}{T^{4}} \frac{1}{\tau^{\prime \prime 3}}\left(a+b \tau^{\prime \prime}+c \tau^{\prime 2}\right)+ & + \\
+ & +\frac{H^{2 n}}{T^{2 n}} P n
\end{aligned}
$$

where

$$
\tau^{\prime \prime}=\left(T^{2}-T_{\mathrm{g}}^{2}\right) / T^{2}
$$


$P n$ is a polynome of degree $2 n+1$ of the variable $1 / \tau^{\prime \prime}$ whose highest order term only is contained in the scaling form :

$q_{\text {sc. }}=\tau^{\prime \prime \beta} F\left(\frac{h^{\prime 2}}{\tau^{\prime \prime \phi}}\right)$ developed in the power of $h^{\prime 2} / \tau^{\prime \prime \phi}$ corrections appear already at second order. $b$ and $c$ are different from zero.
The corrections to scaling appear sooner in the development of $q\left(H^{2}, \tau^{\prime \prime}\right)$ than in the development of $M(H, \tau)$ in the ferromagnetic case where they appear only at order 5. Furthermore the relation between the nonlinear magnetization and the order parameter involve extra non trival mixtures of singular terms and regular terms.

\section{References}

[1] Simpson, M., J. Phys. F 9 (1979) 1377.

Chikazawa, S., Yochunas, Y. G. and Miyako, Y., J. Phys. Soc. Japan 49 (1980) 1276.

[2] Barbara, B., Malozemoff, A. P. and Imry, Y., Phys. Rev. Lett. 47 (1981) 1852.

[3] Monod, P. and Bouchiat, H., J. Physique Lett. 43 (1982) L-45.

[4] Berton, A., Chaussy, J., Odin, J., Rammal, R. and TOUR NIER, R., J. Physique Lett. 43 (1982) L-153.

[5] Omari, R., PréJean, J. J. and Souletie, J., J. Physique 44 (1983) 1069.

[6] Taniguchi, T., Matsuyama, H., Chikazawa, S. and MiYaKo, Y., J. Phys. Soc. Japan 12 (1983) 4323.

[7] Beauvillain, P., Chappert, C., Renard, J. P., J. Physique Lett. 45 (1984) L-665.

Beauvillain, B., Dupas, C., Renard, J. P. and Veillet, P., Phys. Rev. B 29 (1984) 1.

[8] Taniguchi, T., Miyako, Y. and Tholence, J. L., $J$. Phys. Soc. Japan 54 (1985) 220.

[9] Pappa, C. and Hamman, J., J. Physique 46 (1985) 637.

[10] De Dominicis, C., Kondor, I., J. Physique Lett. 45 (1984) L-205.

[11] Binder, K., Z. Phys. B 48 (1982) 319.

[12] Kinzel, W. and Binder, K., Phys. Rev. Lett. 19 (1983) 1509.

[13] BINDER, K. and KinZeL, W., Heidelberg Colloquium on spin glasses (Springer Verlag) 1983.

[14] Binder, K. and Young, A. P., Phys. Rev. B 29 (1984) 2864.

[15] Bhatt, R. N. and Young, A. P., Phys. Rev. Lett. 54 (1985) 924.

[16] Ogielski, A. and Morgenstern, I., Phys. Rev. Lett. 54 (1985) 924.

[17] Sherrington, D. and KirkPatrick, S., Phys. Rev. Lett. 32 (1975) 1792.

[18] De Almeida, J. R. L. and Thouless, D. J., J. Phys. A 11 (1978) 983.

[19] Parisi, G., Phys. Rev. Lett. 43 (1979) 1754 ; Phys. Rev. Lett. 24 (1983) 1948.

[20] Chalupa, J., Solid State Commun. 22 (1977) 315; Solid State Commun. 24 (1977) 424.

[21] Suzuki, M., Prog. Theor. Phys. 58 (1977) 1151.

[22] Toulouse, G. and Gabay, M., J. Physique Lett. 43 (1982) 45.

[23] Souletie, J. and Tholence, J. L., Solid State Commun. 48 (1983) 407.

Fähnle, M. and Souletie, J., J. Phys. C 17 (1984) 469.

[24] GodarT, M. (private communication).

[25] Tholence, J. L., Physica 108B (1981) 1287.

[26] The residual linear contribution is less than $10 \%$ of the total magnetization.
[27] Following the analysis of Omari et al. [5] we have plotted in the insert of figure 12 the quantity $a^{\prime}(T)$ : $a^{\prime}(T)=\chi(T)^{3} a(T)$ versus $\tau^{\prime}=\left(T-T_{\mathrm{g}}\right) / T$ where $\chi(T)=\frac{C}{T}$ for $\underline{A g M n ~} 0.5 \%$. Within this other choice of scaling variables deviations from the $a^{\prime}(T) \propto$ $\tau^{\prime-2}$ like behaviour towards $a^{\prime}(T) \propto \tau^{\prime-3}$ are also observed for $\tau^{\prime}>0.1$.

[28] One could argue indeed that the crossover observed in $P(T)$ and $a(T)$ on figure 12 for the 0.5 at. \% could be suppressed by a shift of $T_{\mathrm{g}}$. The downward temperature shift necessary (more than $4 \%$ ) yields then a transition temperature in a region where strong irreversibilities are observed in the magnetization. Furthermore the modified values $\gamma^{*}=3$ and $\psi^{*}=1$ obtained with this other choice of $T_{\mathrm{g}}$ combined with $\delta=3$ do not verify the scaling relation : $\beta \delta=\beta+\gamma$.

[29] A scaling of the non linear magnetization in AgMn $2.7 \%$ and $9.6 \%$ with values of the critical exponents which are identical to ours has also recently been obtained by $\mathrm{K}$. Baberschke, C. Pappa and R. Wendler (private communication).

[30] For the 20.5 at. \% we took into account of the non zero value of $J_{0}$ (see Sect. 2) the quantity considered is thus $K_{\mathrm{NL}}^{\prime}=\left(M /\left(H+J_{0} M\right)-C / T\right)$ and the magnetic field is replaced by $H^{\prime}=H+J_{0} M$.

[31] Bouchiat, H. and Dartyge, E., J. Physique 43 (1982) 1699.

[32] It has been argued that the R.K.K.Y. interaction is short range because its square (which is the relevant quantity in the spin glass transition) varies like $1 / R^{6}$. A. Bray and Moore, J. Phys. C 15 (1982) 3897.

[33] Chamberlin, R. V., Hardiman, M., Turkevitch, L. A. and OrbaCH, R., Phys. Rev. B 25 (1982) 6720.

[34] Bouchiat, H., Phys. Rev. B 30 (1984) 3963.

[35] Fogle, W. E., Boyer, J. O., Philipps, N. E. and Van Curren, J., Phys. Rev. Lett. 47 (1981) 352.

[36] Mulder, C. A. M., VAN DuYneveldt, A. J. and Mydosh, J. A., Phys. Rev. B 23 (1981) 3.

[37] We recall that in the one dimensional'ferromagnetic Ising model the susceptibility diverges as $\exp (J / T)$ near $T=0$.

[38] Taniguchi, T., Matsuyama, H., Chikazawa, S. and MiYako, Y., J. Phys. Soc. Japan 52 (1983) 4323.

[39] Barbara, B., Malozenoff, A. P. and ImRY, Y., $J$. Appl. Physics 53 (1982) 7662.

[40] Gaunt, P., Ho, S. C. and Williams, G., Phys. Rev. B 23 (1981) 251. 
[41] Fähnle, M. and Egami, T., Solid State Commun. 44 (1982) 533.

[42] Campbell, I. A., de Courtenay, N. and Fert, A., J. Physique Lett. 45 (1984) L-565.

[43] De Courtenay, N., Fert, A. and Campbell, I. A., Phys. Rev. B 30 (1984) 6791.

[44] Fisher, D. S. and Sompolinsky, H., Phys. Rev. Lett. 54 (1985) 1063.

[45] Note that in classical systems critical exponents are higher in Heisenberg systems than in Ising systems.

[46] YeShURUN, Y. and SoMPOLINSKY, H. (preprint, 1985).
[47] Lundgren, L., Svedlindh, P. and Beckman, O., Phys. Rev. B 26 (1982) 3990.

[48] Nordblad, P. and Lundgren, L., J. Phys. Soc. Japan 5A (1985) 1681.

[49] The analysis as in [47] and [48] of the non linear magnetization in a range of field where $M_{\mathrm{NL}} / M<10^{-2}$ yields $\delta \simeq 2$ which is lower than our determination. In such a small field range one may however expect some rounding effects of the transition to take place. 